CERN-TH.6289/91

SISSA $120 / \mathrm{FM} / 91$

\title{
SOLUTIONS OF THE KNIZHNIK - ZAMOLODCHIKOV EQUATION WITH RATIONAL ISOSPINS AND THE REDUCTION TO THE MINIMAL MODELS
}

\author{
P. Furlan \\ Dipartimento di Fisica Teorica dell'Università di Trieste, Italy \\ and Istituto Nazionale di Fisica Nucleare (INFN), Sezione di Trieste, Italy \\ A.Ch. Ganchev* \\ Theory Division, CERN, CH - 1211 Genève 23 \\ and Istituto Nazionale di Fisica Nucleare, Sezione di Trieste \\ R. Paunov* \\ International School for Advanced Studies (SISSA), 34014 Trieste, Italy \\ and \\ V.B. Petkova* \\ Istituto Nazionale di Fisica Nucleare, Sezione di Trieste \\ and International School for Advanced Studies (SISSA), 34014 Trieste
}

\begin{abstract}
In the spirit of the quantum Hamiltonian reduction we establish a relation between the chiral $n$-point functions, as well as the equations governing them, of the $A_{1}^{(1)}$ WZNW conformal theory and the corresponding Virasoro minimal models. The WZNW correlators are described as solutions of the Knizhnik - Zamolodchikov equations with rational levels and isospins. The technical tool exploited are certain relations in twisted cohomology. The results extend to arbitrary level $k+2 \neq 0$ and isospin values of the type $J=j-j^{\prime}(k+2), 2 j, 2 j^{\prime} \in \mathbb{Z}_{+}$.
\end{abstract}

CERN-TH.6289/91

December 1991

\footnotetext{
0* Permanent address: Institute for Nuclear Research and Nuclear Energy, 1784 Sofia, Bulgaria

e-mail: mvxtst::furlan, ganchev@itssissa, paunov@itssissa, petkova@itssissa
} 


\section{Introduction}

The class of conformal field theories introduced by Knizhnik and Zamolodchikov (KZ) [1] is expected to provide non-perturbative solutions of the 2-dimensional WZNW Lagrangian models at renormalization group fixed points. The theory is determined by its infinite dimensional symmetry described by a pair of commuting chiral algebras - each being a semidirect sum of a Virasoro and a Kac-Moody (KM) algebra $\hat{g}$. Because of the holomorphic-antiholomorphic factorization one can focus on one of these chiral algebras. In the KZ theory the Sugawara representation of the Virasoro generators is used, so that the scale dimensions and the Virasoro central charge are expressed in terms of the level $k$ (=central charge) of the KM algebra $\hat{g}$ and the weights $\Lambda$ of the representations of the finite-dimensional subalgebra $g$. The representation of the $L_{-1}$ generator of Virasoro by the Sugawara formula results in the KZ differential equation. This is a linear first-order matrix system of equations for the chiral $n$-point correlation functions of the primary fields

$$
\begin{gathered}
\left(\frac{\partial}{\partial z_{a}}-\frac{1}{(k+h)} \sum_{\substack{b=1 \\
b \neq a}}^{n} \frac{\Omega_{a b}}{\left(z_{a}-z_{b}\right)}\right) W^{(n)}\left(z_{1}, \Lambda_{1} ; \ldots ; z_{n}, \Lambda_{n}\right)=0, \\
a=1,2, \ldots . n
\end{gathered}
$$

where

$$
\Omega_{a b}=q_{\alpha \beta} S_{a}^{\alpha} S_{b}^{\beta}
$$

and $S_{a}^{\alpha}, \alpha=1,2, \ldots, \operatorname{dim} g$, acting on the $a^{\text {th }}$ field, are generators of a representation $C_{\Lambda_{a}}$ of the (complex, semisimple) algebra $g$. The matrix $q$ is the inverse of the Killing-Cartan form and $h$ is the dual Coxeter number. The case most studied is the one described by non-negative integers $k$ and a finite set of irreducible finite-dimensional representations of $g$.

The importance of the WZNW-KZ theories comes from the fact that they generate a large class of rational conformal field theories. In this paper we shall be concerned with the simplest case, the algebra $\hat{g}=\hat{s l}(2, \mathbb{C})$.

The idea of a hidden $\hat{s l}(2)$ symmetry in the Virasoro minimal models (instead of the $\hat{s l}(2) \otimes \hat{s l}(2)$ symmetry of the coset construction) originates from [2]. If we consider rational level and isospins,

$$
\begin{gathered}
J=j-(k+2) j^{\prime} \equiv J_{j, j^{\prime}}, \quad 2 j, 2 j^{\prime} \in \mathbb{Z}_{+}, \\
k+2=p / p^{\prime}
\end{gathered}
$$

$p, p^{\prime}$ - coprime (positive) integers, and $j, j^{\prime}$ - restricted furthermore to

$$
1 \leq 2 j+1 \leq p-1, \quad 1 \leq 2 j^{\prime}+1 \leq p^{\prime}-1,
$$

we can rewrite the central charge and the Kac formula for the conformal dimensions $h\left(j, j^{\prime}\right)$ of the minimal models as

$$
\begin{gathered}
c_{k}=13-6\left(k+2+\frac{1}{k+2}\right), \\
h\left(j, j^{\prime}\right) \equiv h_{J}=\triangle_{J}-J=h_{\underline{J}},
\end{gathered}
$$


where $\underline{J}=k+2-J-1$ and $\triangle_{J}$ are the Sugawara scale dimensions

$$
\triangle_{J}=\frac{J(J+1)}{k+2}
$$

If instead of (1.4) we choose $k+2=-p / p^{\prime}$ we recover the $c>25$ counterpart of the minimal $c<1$ series. More generally for arbitrary $k+2 \neq 0$ and isospins $J_{j, j^{\prime}}$ as in (1.3), the formulae (1.6), (1.7) parametrize the reducible Virasoro Verma modules [3].

Various aspects of KM algebras at rational levels have been studied. In [4] the class of modular representations having characters which close under the action of the modular group was singled out. In the notation used here this class is described (for $g=A_{1}$ ) by the set (1.3), (1.4), (1.5), enlarged to include the points with $2 j^{\prime}+1=p^{\prime}$. In particular the integer level case $k+2=p, 2 J_{a}=2 j_{a} \leq k$, is incorporated with an empty "minimal subset", i.e. $p^{\prime}=1=2 j_{a}^{\prime}+1$. The free field resolution of rational level $A_{1}^{(1)}$ Fock modules was investigated in [0]. The classical Hamiltonian reduction [6] has been carried over to the quantum case on the level of representations of the chiral KM algebra [7]. The Virasoro characters have been recovered as "residues" of $A_{1}^{(1)}$ characters [8] - an example of reduction for genus-one zero-point functions (see also [9] for higher rank generalizations). 1

The full quantum field theory is defined when the correlators are given. Thus after understanding the reduction on the level of the chiral algebra and representations it is natural to study the problem in its fullest, albeit for the simplest example of $g=A_{1}$. In this paper (which is an extended and detailed version of the small note [10]) we address the problem of the construction of the "rational level and isospins WZNW-KZ theory" itself, that is the description of the correlators of rational isospin fields on the plane as solutions of the $\mathrm{KZ}$ equation. The reduction to the corresponding Virasoro minimal theory conformal blocks is straightforward - at the same time, as it will become clear, the relation to the Virasoro models motivates the choice of the solutions. The result holds as well for the larger set described by arbitrary $k+2 \neq 0$ and $J_{j, j^{\prime}}$ as in (1.3).

The finite matrix KZ equation (corresponding to (half)-integer isospins, i.e., $j^{\prime}=0$ in (1.3)) was used in the integer-level case to write down 2-dimensional monodromy invariant 4-point correlation functions [11]. Its simplicity allowed one to investigate directy the monodromy properties of the conformal blocks [12], [13] and to find explicit solutions given by multiple contour integrals [14], [15]. Expressions for the correlators based on the Wakimoto bosonization of the model were also provided [5], 16]. Recently solutions were found for the case of an arbitrary affine KM algebra 17] (see also [18]).

The integrals entering the WZNW correlators in this (finite matrix) case

$$
I_{\mu}^{(s)}(z)=\int_{\Gamma} \Phi_{\lambda}^{(s)}(u, z) \varphi_{\mu}^{(s)}(u, z) d u_{1} \ldots d u_{s}
$$

are of the type first encountered in the minimal models [19], [20], 21]. In (1.9) the number of integrations $s$ is expressed as a linear combination of the isospins $\left\{j_{a}, a=1,2, \ldots, n\right\}$, e.g., $s=$ $j_{1}+j_{2}+\ldots+j_{n-1}-j_{n}$, and $\Phi_{\lambda}^{(s)}$ is a multivalued function of the same structure as the integrand of the thermal (i.e., labelled by $\{h(j, 0)\}$ ) minimal model correlators

$$
\Phi_{\lambda}^{(s)}(u, z)=\prod_{a=1}^{n-1} \prod_{i=1}^{s}\left(u_{i}-z_{a}\right)^{\lambda_{i a}} \prod_{i<j}\left(u_{i}-u_{j}\right)^{\lambda_{i j}} .
$$

\footnotetext{
${ }^{1}$ In [8] a different, parametrization and correspondence from (1.3), (1.7), have been used.
} 
The exponents $\lambda_{i a}, \lambda_{i j}$ are specified by the Coulomb gas representation - see Section 2 for a summary of the minimal and WZNW bosonization technique. The $(n-1)$-vector $\mu=\left(\mu_{1}, \ldots, \mu_{n-1}\right)$ accounts for the isospin degrees of freedom. The factor $\varphi_{\mu}^{(s)}$ is a meromorphic function - it can be chosen to be symmetric with respect to the variables $\left\{u_{i}\right\}$, with simple poles for $u_{i}=z_{a}$

$$
\begin{gathered}
\varphi_{\mu}^{(s)}=\frac{(s-|\mu|) !}{s !}\left(\prod_{i_{1}=1}^{\mu_{1}} \frac{1}{u_{i_{1}}-z_{1}} \prod_{i_{2}=\mu_{1}+1}^{\mu_{1}+\mu_{2}} \frac{1}{u_{i_{2}}-z_{2}} \cdots\right. \\
\left.\ldots \prod_{i_{n-1}=|\mu|-\mu_{n-1}+1}^{|\mu|} \frac{1}{u_{i_{n-1}}-z_{n-1}}+\text { permutations of }\left\{u_{i}\right\}\right), \\
|\mu|=\mu_{1}+\mu_{2}+\ldots \mu_{n-1} .
\end{gathered}
$$

In the WZNW Coulomb gas technique the meromorphic factor (1.11) appears with $|\mu|=s$. It is related to a correlator of the $\beta-\gamma$ system necessary for the bosonization of the KM algebra.

Viewing for a moment the insertion points $z_{a}$ of the primary fields as fixed, the monodromies of the function $\Phi_{\lambda}^{(s)}\left(\left\{u_{i}, z_{a}\right\}\right)$ describe a local coefficient system over the space $X_{s}=\left\{\left(u_{1}, u_{2}, \ldots, u_{s}\right) \in\right.$ $\left.\mathbb{C}^{s} ; u_{i} \neq u_{j}, u_{i} \neq z_{a}\right\}$; the contour $\Gamma$ is a cycle representing an element of the twisted homology $H_{s}\left(X_{s}, \Phi\right)$ [20], 17]. Changing the integration cycle one obtains different solutions of the KZ equation. The differential form $\varphi_{\mu}^{(s)} d u_{1} \wedge \ldots \wedge d u_{s}$ can be viewed as an element of the dual twisted cohomology $H^{s}\left(X_{s}, \nabla_{\Phi}\right), \nabla_{\Phi}=\nabla-\nabla(\log \Phi)$. The integrals $I_{\mu}$ are examples of generalized hypergeometric integrals studied recently [22], [23].

The solutions of the type (1.9) are valid for arbitrary $k+2 \neq 0$ and in particular for rational $k$ they describe the "thermal" correlators. We will refer in general to a spin $J=j-j^{\prime}(k+2)$ with $j^{\prime}=0$ as a thermal spin while the one with $j=0$ we will call a quasithermal spin. In both cases the corresponding minimal model integrals are obtained using one type of screening charges and the factor $\Phi_{\lambda}^{(s)}$ in the thermal WZNW correlator (1.9) coincides with the integrand of the corresponding minimal block. 2 One can expect that the generalization of (1.9) for arbitrary rational spins will involve the general non-thermal integrand. The problem is to generalize the factors $\varphi_{\mu}$. We recall that in the $A_{1}^{(1)}$ Coulomb gas there are two solutions for the screening currents. In the description of the free field resolution [5] one can do with only one type of screening charges. On the other hand, to define a general correlator with rational spins (1.3) one is forced to consider both types of screening charges. This lies at the heart of the problem since the second charge involves a non-integer power $(-(k+2))$ of the bosonic field $\beta$. If one defines [24] the correlator of rational spin fields by analytic continuation in $J_{a}$ and $k$ of the bosonization formulae, valid for integer $2 J_{a}$ and $k$, the factor $\varphi-$ related to an expectation value of the $\beta-\gamma$ system - is no longer a meromorphic function. Thus effectively one has changed the local coefficient system defined by $\Phi$. This may change the fusion rules if compared with the fusion rules of the corresponding minimal model, irrespectively of the values of $p, p^{\prime}$, as happens in the simple example investigated in [24]. Hence the direct connection between the correlators of the two types of models is eventually lost.

The difficulty is related to the fact that we have to deal with infinite dimensional representations of the isospin algebra $s l_{I}(2, \mathscr{C})$ when we allow non-integer $2 J$. Putting the isospin and the coordinate

\footnotetext{
${ }^{2}$ In a weaker sense we will call "thermal" (or "quasithermal") the integrals corresponding to only one type of screening charges. Thus, e.g., the "thermal" integrals (1.9) can accomodate arbitrary $\left\{J_{a}\right\}$, restricted only by the condition that $s=J_{1} \ldots+J_{n-1}-J_{n}$ is a non-negative integer.
} 
$s l(2, \mathbb{C})$ symmetry on equal footing one can realize the generators $S^{0}, S^{+}, S^{-}$of $s l_{I}(2, \mathbb{C})$ as differential operators with respect to a second complex variable $x$, i.e.,

$$
S^{0}=2 x \partial_{x}-2 J, \quad S^{-}=-\partial_{x}, \quad S^{+}=x^{2} \partial_{x}-2 J x .
$$

If in these differential operators we replace $(x, J)$ by $(z,-\triangle)$ we obtain the generators $2 L_{0},-L_{-1}$, $L_{1}$ of the $\operatorname{sl}(2, \mathbb{C})$ subalgebra of Virasoro. This realization has been used in [11] in the (half)-integer isospin case $J=j$ where there exists an invariant finite dimensional subspace of fields $\Psi^{J}(x, z)$, spanned by all polynomials of $x$ of highest degree $2 J$. (In a Minkowski space framework the variables $x$ and $z$ are real and $S^{\alpha}$ (or $L_{\alpha}$ ), $\alpha= \pm 1,0$, represent the generators of an induced representation of the group $S L\left(2, \mathbb{R}\right.$ ) labelled by $J$ (or $-\triangle$ ).) The (left) action of the generators $X_{n}^{\alpha}$ of the KM algebra $\hat{g}$ on the primary fields $\Psi^{J}(x, z)$ reduces to $S^{\alpha}$, as defined in (1.12), times $z^{n}$. The field $\Psi^{J}(x, z)$ can be treated as a highest (or lowest) weight state with respect to a right action of $\hat{g}$, thus generating a module of descendants. (See [11] for explicit formulae which carry over to arbitrary values of $J$.)

Inserting the expression (1.12) for the generators in the KZ system (1.1) we can look for solutions of the resulting equations in the space of all $n$-point invariants with respect to both $\operatorname{sl}(2, \mathscr{C})$ algebras for arbitrary values of the isospins. The starting point of our work has been the following simple observation. The 2- and 3-point chiral functions are given by the factorized expressions

$$
\begin{aligned}
& W^{(2)}\left(x_{1}, z_{1}, J ; x_{2}, z_{2}, J\right)=\left(x_{12}\right)^{2 J}\left(z_{12}\right)^{-2 \Delta_{J}}, \\
& W^{(3)}\left(x_{1}, z_{1}, J_{1} ; \ldots ; x_{3}, z_{3}, J_{3} ;\right)=C_{J_{1} J_{2} J_{3}} \frac{x_{12}^{J_{1}+J_{2}-J_{3}} x_{23}^{J_{2}+J_{3}-J_{1}} x_{31}^{J_{1}+J_{3}-J_{2}}}{z_{12}^{\Delta_{J_{1}}+\Delta_{J_{2}}-\Delta_{J_{3}}} z_{23}^{\Delta_{J_{2}}+\Delta_{J_{3}}-\Delta_{J_{1}}} z_{31}^{\Delta_{J_{1}}+\Delta_{J_{3}}-\Delta_{J_{2}}}} .
\end{aligned}
$$

Setting $x_{a}=z_{a}$ and using (1.7) we immediately obtain the respective functions of the minimal models. This simple factorization is no longer valid for higher $n$-point functions. Instead we can try to find solutions for the correlators in terms of power series of $\left(x_{a}-z_{a}\right)$. For example, for the 4-point function we can write, using the projective invariance,

$$
W^{(4)}\left(\left\{x_{a}, z_{a}\right\}\right)=\text { prefactor } \sum_{t=0}(\underline{x}-\underline{z})^{t} C_{t}(\underline{z})
$$

where $\underline{x}$ and $\underline{z}$ are anharmonic ratios.

The KZ equation for the 4-point function translates into an (infinite in general) matrix differential equation for the coefficient functions $C_{t}$ in (1.15). It is a generalization of the finite $\mathrm{KZ}-\mathrm{ZF}$ system (for Knizhnik, Zamolodchikov, Fateev) written down in the integer level case. It is instructive to start first with the chiral solutions of the finite KZ-ZF system. In Section 3 we describe the set of integrals serving as coefficients in the $(x-z)$-expansion of the $n$-point chiral correlators in the thermal case. In particular for $n=4$ these integrals $\left\{C_{t}(\underline{z})=B_{t} I_{t}^{(s)}(\underline{z})\right\}$ are, up to the numerical coefficients $B_{t}$, of the type in (1.9), (1.10), (1.11), with $\mu=(0, t, 0), t=0,1, \ldots, s$. These solutions are equivalent to the representations of the $n$-point functions coming from bosonization, [5], [16], or other related representations studied in the literature [15]. The latter can be written as $x$-expansions and they involve $\varphi_{\mu}^{(s)}$ with $|\mu|=s$. The tool used to prove the equivalence of the various integral representations in the thermal case is a set of linear relations, derived by Aomoto [22] in the study of the generalized hypergeometric integrals and the twisted cohomology groups 
related to them. The $(x-z)$ - expansion can be interpreted as providing a new basis in the top (i.e., the $s^{\text {th }}$ ) twisted cohomology which includes $\varphi_{0}^{(s)} \simeq 1$ as an element or - upon integration the integral $I_{0}^{(s)}$, which coincides with the corresponding minimal model integral. This basis is also suitable for the reduction of the KZ- ZF system studied in Section 3.2. Indeed the integrals in the $(x-z)$ - expansion (1.15) can be written as

$$
\gamma_{t} I_{t}^{(s)}(z)=\mathcal{L}_{t}(z, \partial) I_{0}^{(s)}(z), \quad t=1,2, \ldots
$$

with $\mathcal{L}_{t}$ a differential operator of order $t$ and $\gamma_{t}-$ a numerical coefficient (see formulae (3.2.3), (3.2.5)). Since the KZ-ZF in the thermal case is a finite system, for a certain $t$ the left-hand side of (1.16) vanishes and we obtain the corresponding BPZ differential equation for the minimal model correlators describing the decoupling of the Virasoro singular vectors [25]. Thus in the thermal case we have two theories that are independently well defined - the WZNW having correlators that are solutions of a finite KZ - ZF system and the corresponding minimal model. Taking $x \rightarrow z$ we get a reduction of the first to the second. Vice versa, given the minimal blocks we can recover the WZNW correlations according to (1.16). Note that the situation in the integer level case, which does not have a minimal model analogue, is somewhat different. Depending on the specific combinations of the spins, the series (1.15) appears to be effectively truncated from below 11] so that the limit $x_{a} \rightarrow z_{a}$ is trivial.

In the non-thermal case the WZNW conformal theory is not uniquely defined. The KZ - ZF system is infinite, reflecting the fact that for non-thermal spins $J$ the representations of $g$ are infinite-dimensional. In Section 2.2 we briefly discuss the various solutions coming from the analytic continuation of the bosonization technique. Motivated by the consideration in the thermal case we generalize in Section 4.1 the $(x-z)$-expansion finding explicit solutions for the coefficients. Now the two types of solutions are no longer equivalent. While the former involves, as mentioned, multivalued functions $\varphi_{\mu},|\mu|=s-(k+2) s^{\prime}$, the latter is described by "meromorphic" factors $\varphi_{\mu}^{\left(s, s^{\prime}\right)}, \mu_{a} \in \mathbb{Z}_{+}$, generalizing (1.11). The hard part is to find these non-thermal meromorphic factors (see formulae (4.1.3), (4.1.1)). The corresponding integrals satisfy a system of linear and recursion relations which have a cohomological interpretation and generalize the ones in the thermal case. All this highly technical part is put in a separate section - Section 5. The solutions of the KZ-ZF infinite system described in Section 4.1 are such that for generic spins they reduce in the limit $x \rightarrow z$ to the minimal correlations. One can look at the resulting WZNW theory, described by an infinite set of integrals $\left\{I_{\mu}^{\left(s, s^{\prime}\right)},|\mu|=0,1, \ldots\right\}$, as being "generated" uniquely by the corresponding minimal one $I_{0}^{\left(s, s^{\prime}\right)}$. The correlators in this theory have the same braiding properties, under simultaneous change of $x$ and $z$, and hence the same fusion rules as the corresponding minimal Dotsenko-Fateev (DF) blocks. These "meromorphic" solutions can be viewed as analogues of the "odd" combinations of KM characters in [8] (labelled by $J$ and $\underline{J}$ ) which transform with the minimal model modular matrix.

The rationality of the factor $\varphi_{\mu}^{\left(s, s^{\prime}\right)}$, also ensures the actual truncation of the infinite KZ $\mathrm{ZF}$ matrix system of equations to a finite system, which can furthermore be reduced to a BPZ type equation for the minimal integrals. The origin of this truncation is the existence of additional linear relations for the integrals which reflects the fact that any rational form can be expressed up to an exact form in terms of a finite basis in the relevant twisted cohomology [22]. Section 4.3 contains a preliminary discussion of this point which deserves further study. In Section 4.2 and in the Appendix we describe, using the example of $n=4$, an explicit algorithm to obtain these relations in the quasithermal case. It is based on a duality property of the WZNW models with $k+2,\left\{J_{a}\right\}$ and with $\hat{k}+2=1 /(k+2),\left\{\hat{J}_{a}=-J_{a} /(k+2)\right\}$ - both generated by the same minimal 
correlation functions. On the other hand the additional relations, and hence the truncation, can be obtained equivalently taking into account one of the algebraic null vector decoupling equations, originating from singular vectors of the $A_{1}^{(1)}$ Verma modules. This is discussed and illustrated in examples in Section 4.3. Section 6 contains a discussion of the open problems.

\section{Free field realizations of the minimal and $A_{1}^{(1)}-$ models}

\section{$2.1 \quad$ Minimal models}

The free field realization of the minimal models [19] utilizes a free bosonic chiral field $\varphi(z)$ with a logarithmic 2-point function and a modified energy momentum-tensor reproducing the central charge (1.6). A primary field $\Psi^{h\left(j, j^{\prime}\right)}(z)$ of conformal dimension $h\left(j, j^{\prime}\right)$ given by (1.7) is represented by a vertex operator

$$
V_{\alpha}(z)=: e^{i \alpha \varphi(z)}:
$$

of charge

$$
\alpha=\alpha\left(j, j^{\prime}\right)=-j \alpha_{-}-j^{\prime} \alpha_{+}=-J \alpha_{-} \equiv \alpha_{J}, \quad \text { or } \quad \alpha=2 \alpha_{0}-\alpha\left(j, j^{\prime}\right)=\alpha_{\underline{J}},
$$

where

$$
2 \alpha_{0}=\alpha_{+}+\alpha_{-}, \quad \alpha_{ \pm}= \pm(k+2)^{ \pm 1 / 2} .
$$

The vertex operators in a correlator must satisfy a charge conservation condition shifted by the charge $-2 \alpha_{0}$ at infinity. In order to be able to write non-trivial correlators one needs (non-local) fields of non-zero charge and zero conformal dimension so that the insertion of such fields inside correlators could fit the charge balance without changing the conformal properties. These are the screening charges - contour integrals of the conformal dimension 1 screening currents

$$
V_{ \pm}(z)=V_{\alpha_{ \pm}}(z)
$$

The correlators of $n$ primary fields $\Psi^{h_{a}}\left(z_{a}\right), h_{a}=h\left(j_{a}, j_{a}^{\prime}\right), a=1, \ldots, n$ are thus represented by a multiple contour integral

$$
\begin{aligned}
& \mathcal{S}_{\Gamma}^{(n)}\left(z_{1}, \ldots, z_{n}\right)=\left\langle 0\left|\Psi^{h_{1}}\left(z_{1}\right) \Psi^{h_{2}}\left(z_{2}\right) \ldots \Psi^{h_{n}}\left(z_{n}\right)\right| 0\right\rangle_{\Gamma}= \\
& \int_{\Gamma} d u_{1} \ldots d u_{s} d v_{1} \ldots d v_{s^{\prime}}\left\langle V_{\alpha_{1}}\left(z_{1}\right) \ldots V_{\alpha_{n}}\left(z_{n}\right) V_{-}\left(u_{1}\right) \ldots V_{-}\left(u_{s}\right) V_{+}\left(v_{1}\right) \ldots V_{+}\left(v_{s^{\prime}}\right)\right\rangle_{\alpha_{0}} .
\end{aligned}
$$

Without lack of generality we can choose $\alpha_{a}=\alpha\left(j_{a}, j_{a}^{\prime}\right), a=1,2, \ldots, n-1$ and $\alpha_{n}=2 \alpha_{0}-\alpha\left(j_{n}, j_{n}^{\prime}\right)$ so that charge neutrality implies

$$
s=j_{1}+j_{2}+\ldots+j_{n-1}-j_{n}, \quad s^{\prime}=j_{1}^{\prime}+j_{2}^{\prime}+\ldots+j_{n-1}^{\prime}-j_{n}^{\prime} .
$$

Using the projective invariance of the conformal blocks, 2.1.5 can be written as

$$
\mathcal{S}_{\Gamma}^{(n)}\left(z_{1}, \ldots, z_{n}\right)=f\left(\left\{z_{a} ; h_{a}\right\}_{a=1}^{n}\right) \prod_{1 \leq a<b \leq n-1}\left(\underline{z}_{a}-\underline{z}_{b}\right)^{2 J_{a} J_{b} /(k+2)} I_{\Gamma}^{\left(s, s^{\prime}\right)}(\underline{z}),
$$

where

$$
\underline{z}_{a}=\frac{z_{1 a} z_{n-1, n}}{z_{1, n-1} z_{a n}}
$$


for $a=2, \ldots, n-2$, are the anharmonic ratios (the moduli of the $n$-punctured sphere) and the prefactor in (2.1.7) is

$$
f\left(\left\{z_{a} ; h_{a}\right\}_{a=1}^{n}\right)=\prod_{a=1}^{n-1}\left(z_{a}-z_{n}\right)^{-2 h_{a}}\left(\frac{\left(z_{1}-z_{n}\right)\left(z_{n-1}-z_{n}\right)}{\left(z_{1}-z_{n-1}\right)}\right)^{\sum_{a=1}^{n-1} h_{a}-h_{n}} .
$$

The data in (2.1.5) or (2.1.7) consist of the integration contours $\Gamma$ and the spins $J_{a}$ attached to the punctures $z_{a}$ (or $\underline{z}_{a}$ ). For short, we will denote the respective $(n-1)$ - vectors by $z=\left(z_{a}\right)$ (or $\underline{z}=\left(\underline{z}_{a}\right)$ ) and $J=\left(J_{a}\right), a=1, \ldots, n-1$. Instead of $J_{n}$ we will specify $\left(s, s^{\prime}\right)$ or equivalently $S=s-s^{\prime} \alpha_{+}^{2}$. Most of the time we will skip indicating part of the data when there will be no danger of confusion.

The computation of the integrand in $(2.1 .5)$ gives

$$
I_{\Gamma}^{\left(s, s^{\prime}\right)}(\underline{z})=\int_{\Gamma} d u_{1} \ldots d u_{s} d v_{1} \ldots d v_{s^{\prime}} \Phi_{J}^{\left(s, s^{\prime} ; \alpha_{-}^{2}\right)}\left(u_{i}, v_{i^{\prime}} ; \underline{z}_{a}\right)
$$

where the multivalued function $\Phi$ is given by (cf. (1.10) )

$$
\Phi_{J}^{\left(s, s^{\prime} ; \nu\right)}\left(u_{i}, v_{i^{\prime}} ; z_{a}\right)=\Phi_{J}^{(s ; \nu)}\left(u_{i} ; z_{a}\right) \Phi_{-\nu J}^{\left(s^{\prime} ; 1 / \nu\right)}\left(v_{i^{\prime}} ; z_{a}\right) \prod_{i=1}^{s} \prod_{i^{\prime}=1}^{s^{\prime}} \frac{1}{\left(u_{i}-v_{i^{\prime}}\right)^{2}},
$$

and

$$
\Phi_{J}^{(s ; \nu)}\left(u_{i} ; z_{a}\right)=\prod_{i<j}^{s}\left(u_{i}-u_{j}\right)^{2 \nu} \prod_{i=1}^{s} \prod_{a=1}^{n-1}\left(u_{i}-z_{a}\right)^{-2 J_{a} \nu} .
$$

Recall that we denote $J_{a}=j_{a}-j_{a}^{\prime} \alpha_{+}^{2}$, thus for $\nu=\alpha_{-}^{2}$ we have $-\nu J_{a}=j_{a}^{\prime}-j_{a} \alpha_{-}^{2}$.

Making different choices for the integration contours $\Gamma$ we can obtain a basis in the space of $n$-point functions. One possibility to get a "basic" set is to use the notion of a chiral vertex operator ${ }_{h_{1}} \Psi_{h_{2}}^{h}(z)=P_{h_{1}} \Psi^{h}(z) P_{h_{2}}$, where $P_{h}$ is a projector on the Virasoro representation of highest weight $h$. These operators can be realized as screened vertex operators [21] with screening charges integrated along a double loop (see also [26]). Inserting projectors between primary fields we obtain an $n$-point conformal block with specified spins in the intermediate channels labelled by a vector $K=\left(K_{a}\right)$ (with $K_{a}=k_{a}-k_{a}^{\prime} \alpha_{+}^{2}$ ) such that $K_{1}=J_{1}, K_{n-1}=J_{n}$. The corresponding contour $\Gamma_{K}$ is a configuration of nested double loops (i.e. the $i^{\text {th }}$ contour consists of a loop around $z_{i+1}$ and a loop encircling all $\left.z_{1}, z_{2}, \ldots, z_{i}\right)$. We will call "admissible" the set of such contours when the intermediate channels $\left(K_{a}\right)$ are consistent with the fusion rules, i.e., for every triple $\left(K_{a-1}, J_{a}, K_{a}\right)$, $a=2, \ldots, n-1$ the fusion rules are satisfied. Another such choice is provided by a subset of the contours connecting the singular points [19]. The minimal models fusion rules multiplicities $N_{h_{2} h_{1}}^{h}=\left(N^{h}\right)_{h_{2} h_{1}}$ (= the number of non-zero chiral vertex operators $h_{1} \Psi_{h_{2}}^{h}$ ) have the factorized form

$$
N_{h\left(k_{2}, k_{2}^{\prime}\right) h\left(k_{1}, k_{1}^{\prime}\right)}^{h\left(j, j^{\prime}\right.}=N_{k_{2} k_{1}}^{j} N_{k_{2}^{\prime} k_{1}^{\prime}}^{j^{\prime}}
$$

where $N_{k_{2} k_{1}}^{j}$ is symmetric with respect to all indices and $N_{k_{2} k_{1}}^{j}=1$ if and only if

$$
\left|k_{1}-k_{2}\right| \leq j \leq \min \left(k_{1}+k_{2}, p-2-k_{1}-k_{2}\right),
$$

otherwise $N_{k_{2} k_{1}}^{j}=0$, and analogously for the primed spins. For fixed $J_{1}, \ldots, J_{n}$ the dimension of the space of $n$-point correlators with $\Gamma_{K}$ is thus

$$
\left(N^{h\left(J_{n-1}\right)} \ldots N^{h\left(J_{3}\right)} N^{h\left(J_{2}\right)}\right)_{h\left(J_{n}\right) h\left(J_{1}\right)} .
$$


This bears some features in common with the set of $s l(2, \mathbb{C})$ - invariant tensors. Actually the relevant structure is related to a deformation of this algebra, the quantum universal enveloping algebra $U_{q}(\operatorname{sl}(2, \mathscr{C}))$ when the deformation parameter is $q=\exp \left(2 \pi i p / p^{\prime}\right)$ or $q^{\prime}=\exp \left(2 \pi i p^{\prime} / p\right)$ (see e.g. [27] and references therein).

The formalization of the above description is in terms of twisted homology, i.e., homology on the space $X_{s+s^{\prime}}$ of the positions $u_{i}, i=1, \ldots, s$ and $v_{i^{\prime}}, i^{\prime}=1, \ldots, s^{\prime}$ of the screening charges (with no two of the points $z_{a}, u_{i}, v_{i^{\prime}}$ coinciding) with coefficients in the local system defined by the multivalued function $\Phi$. The general statement is that the integration contour $\Gamma$ is a cycle in the top twisted homology and, as $\Gamma$ runs over a basis in homology, the integrals $\int_{\Gamma} \Phi$ give a basis in the space of correlators.

These statements actually need a lot of refining in the case of the generalized hypergeometric integrals (2.1.10) which appear in the minimal theories. In fact there are quite a few points that still await a precise mathematical treatment. These questions do not constitute the core of our discussion and we will be content with several remarks.

The appropriate homology depends on the type of contours. When the exponents in (2.1.11) are rational numbers, the usual assumptions of "non-resonance" of these exponents [22], [23] are not valid in general and the dimension of the "homology of double loops" can be different from the dimension of the respective homology of contours which turn on singular points. Accordingly, depending on the choice of the contours, the vertex representation provides the conformal blocks of (at least) two essentially different types of models. The minimal one - in which the symmetry $\alpha_{J} \rightarrow 2 \alpha_{0}-\alpha_{J}$ is preserved - is described by an admissible set of contours. The second leads to "unphysical" (i.e., not in the domain (1.5) contributions and it admits an interpretation in terms of quantum group modules. This is also related to the peculiarities of the quantum group representation theory when the parameter $q$ is a root of unity.

Finally let us mention one more point that deserves a rigorous treatment. The general nonthermal multivalued function (2.1.11) involves integer exponents and has a peculiar factorised form suggesting that we should consider a factor of homology - identifying cycles that differ by interchanging $u$ and $v$ contours. Thus this factor homology factorizes into $u$ cycles times $v$ cycles (in agreement with the results of [19]) and this leads to the factorization of the fusion rules (2.1.13).

\section{$2.2 A_{1}^{(1)}-$ models}

The free field realization of these models involves the free field $\varphi(z)$ and a pair of generalized free bosonic fields $(\beta, \gamma)$ with a $2-$ point function

$$
\left\langle\beta\left(z_{1}\right) \gamma\left(z_{2}\right)\right\rangle=\frac{1}{z_{1}-z_{2}} .
$$

The chiral algebra is expressed in terms of this triple of bosonic fields.

Let all $2 J=2 j$ be non-negative integers. The primary fields $\Psi^{J}(x, z)$ are then given by the polynomials

$$
\Psi^{j}(x, z)=\sum_{m=-j}^{j}\left(\begin{array}{c}
2 j \\
j-m
\end{array}\right) x^{j+m} \Psi_{m}^{j}(z),
$$

where the coefficients $\Psi_{m}^{j}(z)$ can be represented as

$$
\gamma^{j-m}(z) V_{\alpha_{j}}(z)
$$


using the vertex operator (2.1.1) of charge $\alpha_{j}=-j \alpha_{-}$. The screening current is given by

$$
\Psi_{-}(u)=\beta(u) V_{-}(u)
$$

with $V_{-}(u)$ defined in (2.1.4).

Another representation for the operator $\Psi_{m}^{j}(z)$ is provided using the vertex operator of conjugate charge $2 \alpha_{0}-\alpha_{j}$. It involves also the second bosonic field $\beta$ and it looks simple only for the state with $m=j$. Namely, within the correlation functions we can represent $\Psi_{j}^{j}(z)$ by

$$
\beta^{2 j}(z) V_{2 \alpha_{0}-\alpha_{j}}(z)
$$

Exploiting the isospin projective invariance of the correlators we can always put one of the fields at $x=\infty$, so that only the component with $m=j$ survives in $x^{-2 j} \Psi^{j}(x, z)$ in this limit.

We stress that the representation (2.2.5) is consistent only for the field averages. They are computed using the factorization of the $\varphi$-and $\beta, \gamma$-dependent contributions; see, e.g., [24] for a detailed description of the bosonization in the operator framework. Thus the correlator of $n$ fields $\Psi_{m_{a}}^{j_{a}}\left(z_{a}\right), a=1, \ldots, n$ is expressed by the average of an equal number of $\beta$ and $\gamma$ fields, times a vertex correlator in the presence of a background charge $-2 \alpha_{0}$ of the type encountered in the minimal theory, namely

$$
\begin{gathered}
\left\langle 0\left|\Psi_{m_{1}}^{j_{1}}\left(z_{1}\right) \ldots \Psi_{j_{n}}^{j_{n}}\left(z_{n}\right)\right| 0\right\rangle_{\Gamma}=\int_{\Gamma} d u_{1} \ldots d u_{s}\left\langle\gamma^{\tau_{1}}\left(z_{1}\right) \ldots \gamma^{\tau_{n-1}}\left(z_{n-1}\right) \beta^{2 j_{n}}\left(z_{n}\right) \beta\left(u_{1}\right) \ldots \beta\left(u_{s}\right)\right\rangle \\
\cdot\left\langle V_{\alpha_{j_{1}}}\left(z_{1}\right) \ldots V_{\alpha_{j_{n-1}}}\left(z_{n-1}\right) V_{2 \alpha_{0}-\alpha_{j_{n}}}\left(z_{n}\right) V_{-}\left(u_{1}\right) \ldots V_{-}\left(u_{s}\right)\right\rangle_{\alpha_{0}},
\end{gathered}
$$

where

$$
\tau_{a}=j_{a}-m_{a}, a=1, \ldots, n-1 ;|\tau|=\tau_{1}+\tau_{2}+\ldots+\tau_{n-1}, \quad|\tau|=2 j_{n}+s,
$$

and as above the charge conservation condition implies that $s=j_{1}+j_{2}+\ldots+j_{n-1}-j_{n}$, so that $m_{1}+m_{2}+\ldots+m_{n-1}+j_{n}=0$.

Using the projective invariance we can write the $n$-point function of the fields $\Psi^{J}(x, z)$ as

$$
W^{(n)}\left(\left\{x_{a}, z_{a}, J_{a}\right\}_{a=1}^{n}\right)=f\left(\left\{x_{a},-J_{a}\right\}_{a=1}^{n}\right) f\left(\left\{z_{a}, \triangle_{a}\right\}_{a=1}^{n}\right)\left(2 J_{n}\right) ! \prod_{1 \leq a<b \leq n-1} \underline{z}_{a b}^{2 J_{a} J_{b} /(k+2)} G_{J}^{(n)}(\underline{x}, \underline{z})
$$

where $f$ is the prefactor defined in (2.1.9). Most of the time we will work with correlators in which the last point is sent to infinity

$$
U^{(n)}(x, z) \equiv \frac{1}{\left(2 J_{n}\right) !} \lim _{\substack{x_{n \rightarrow \infty} \\ z_{n} \rightarrow \infty}} x_{n}^{-2 J_{n}} z_{n}^{2 \triangle_{n}} W^{(n)}\left(\left\{x_{a}, z_{a}, J_{a}\right\}_{a=1}^{n}\right)=\prod_{1 \leq b<a \leq n-1} z_{a b}^{2 J_{a} J_{b} /(k+2)} G_{J}^{(n)}(x, z) .
$$

For thermal isospins $J_{a}=j_{a}(2.2 .2)$ and $(2.2 .6)$ give

$$
G_{j}^{(n)}(x, z)=\sum_{|\tau|=s+2 j_{n}} \prod_{a=1}^{n-1}\left(\begin{array}{c}
2 j_{a} \\
\tau_{a}
\end{array}\right) x_{a}^{2 j_{a}-\tau_{a}} \mathcal{G}_{\left(\tau_{1}, \ldots, \tau_{n-1}\right)}(z)
$$




$$
\mathcal{G}_{\left(\tau_{1}, \ldots, \tau_{n-1}\right)}(z)=\int_{\Gamma} d u_{1} \ldots d u_{s} \Phi_{J}^{(s ; \nu)}\left(u_{i} ; z_{a}\right) \phi_{\left\{\tau_{1}, \ldots, \tau_{n-1}\right\}}\left(u_{i} ; z_{a}\right)
$$

The correlator $\phi_{\tau}$ of the generalized free fields $\beta, \gamma$ is computed by Wick contractions using the 2-point kernel (2.2.1). Here we shall write down the explicit expression for a more general bosonic correlator with arbitrary non-negative integer powers of the $\beta, \gamma$ fields, which will be useful in what follows. To do that let us first introduce some notation.

The indices $a, b(=1, \ldots, n-1)$ will be reserved for the positions of the vertex operators $z_{a}, z_{b}$ and the indices $i, j(=1, \ldots, s)$ for the coordinates $u_{i}, u_{j}$ of the screening currents. The convention will be, if not indicated otherwise, that sums and products in $a$ or $b$ will run over $1, \ldots, n-1$, while those in $i$ or $j$-over $1, \ldots, s$. For an $(n-1)$-vector of non-negative integers we will use the notation $\mu=\left(\mu_{a}\right)$ (also $\tau$ and $\sigma$ ) and the sum of its elements $|\mu|=\sum_{a} \mu_{a}$.

Let $\mu=\left(\mu_{a}\right)$ and $l=\left(l_{i}\right)$ be $(n-1)$ - and $s$-vectors of non-negative integers and such that $|\mu|=|l|$. Consider a rectangular matrix $A=\left(A_{i a}\right)$ with integer valued entries $A_{i a}=0,1,2, \ldots$ and such that

$$
\sum_{a=1}^{n-1} A_{i a}=l_{i}
$$

and

$$
\sum_{i=1}^{s} A_{i a}=\mu_{a}
$$

Denote

$$
\varphi_{A}^{l}(u, z)=\frac{\prod_{a} \mu_{a} !}{\prod_{i, a} A_{i a} !\left(u_{i}-z_{a}\right)^{A_{i a}}}
$$

and

$$
\varphi_{\mu}^{l}(u, z)=\sum_{A} \varphi_{A}^{l}(u, z)=\sum_{A} \frac{\prod_{a} \mu_{a} !}{\prod_{i, a} A_{i a} !\left(u_{i}-z_{a}\right)^{A_{i a}}}, \quad|\mu|=|l|,
$$

where the sum is over all $\left\{A_{i a}\right\}$ satisfying (2.2.12), (2.2.13).

Let $\tau=\left(\tau_{1}, \ldots, \tau_{n-1}\right)$ be a $(n-1)$ - vector with non-negative integer entries. Using the above notation, the bosonic correlator

$$
\phi_{\tau}^{l}\left(u_{i} ; z_{a}\right)=\frac{1}{\prod_{i=1}^{s} l_{i} !\left(2 j_{n}\right) !} \lim _{z_{n} \rightarrow \infty}\left(z_{n}\right)^{2 j_{n}}\left\langle 0\left|\gamma^{\tau_{1}}\left(z_{1}\right) \ldots \gamma^{\tau_{n-1}}\left(z_{n-1}\right) \beta^{2 j_{n}}\left(z_{n}\right) \beta^{l_{1}}\left(u_{1}\right) \ldots \beta^{l_{s}}\left(u_{s}\right)\right| 0\right\rangle
$$

can be written as

$$
\phi_{\tau}^{l}=\sum_{\mu:|\mu|=|l|} \prod_{a}\left(\begin{array}{c}
\tau_{a} \\
\mu_{a}
\end{array}\right) \varphi_{\mu}^{l} .
$$

In the thermal case $l=\rho \equiv(1,1, \ldots, 1)$, so that $|l|=s$ and $A_{i a}=0,1$. Hence the integrand in (2.2.11) is a modification with a meromorphic factor of the minimal thermal multivalued function (2.1.12). Multiplying (2.2.16) (for $l=\rho$ ) by the contribution of the vertex operators and integrating, we get for the thermal conformal blocks (2.2.11)

$$
\begin{aligned}
& \mathcal{G}_{\left(\tau_{1}, \ldots, \tau_{n-1}\right)}(z)=\sum_{\mu:|\mu|=s} \prod_{a}\left(\begin{array}{c}
\tau_{a} \\
\mu_{a}
\end{array}\right) I_{\mu \Gamma}^{(s)}(z), \\
& I_{\mu \Gamma}^{(s)}=\int_{\Gamma} d u_{1} \ldots d u_{s} \Phi_{j}^{(s ; \nu)}(u, z) \varphi_{\mu}(u, z) .
\end{aligned}
$$


Note that in that case $\varphi_{\mu}\left(\equiv \varphi_{\mu}^{\rho}\right)$ itself can be written as a $(\beta, \gamma)$ correlator

$$
\varphi_{\mu}\left(\left\{u_{i}, z_{a}\right\}\right)=(-1)^{s}\left\langle\gamma\left(u_{1}\right) \ldots \gamma\left(u_{s}\right) \beta^{\mu_{1}}\left(z_{1}\right) \ldots \beta^{\mu_{n-1}}\left(z_{n-1}\right)\right\rangle
$$

which coincides with $\varphi_{\mu}^{(s)}$ in (1.11), taken for $|\mu|=s$.

The integral representation (2.2.17) can be extended to rational isospins $J_{a}$ if $s^{\prime}=j_{1}^{\prime}+j_{2}^{\prime}+$ $\ldots+j_{n-1}^{\prime}-j_{n}^{\prime}=0$, so that $J_{1}+J_{2}+\ldots+J_{n-1}-J_{n}=s$. However, to describe the correlators for arbitrary rational spins $J_{a}$ one needs a second screening charge operator, which is provided by

$$
\Psi_{+}(v)=\beta^{K}(v) V_{+}(v) ; \quad K=-(k+2) .
$$

Now the natural idea [24] is to compute the correlations using the two types of screening currents and the vertex operators (2.2.3), (2.2.5) for general isospins, i.e., replacing $j_{a}$ by $J_{a}$ and assuming first that all $J_{a}$ and $K=-(k+2)$ are integers. Then we have to continue in some way the final result. The equality of the number of $\beta$ and $\gamma$ fields implies the condition

$$
|\tau|=2 J_{n}+S, S=s+s^{\prime} K ; \quad s^{\left({ }^{\prime}\right)}=j_{1}^{\left({ }^{\prime}\right)}+j_{2}^{\left({ }^{\prime}\right)}+\ldots j_{n-1}^{\left({ }^{\prime}\right)}-j_{n}^{\left({ }^{\prime}\right)} .
$$

The correlator of the vertex operators gives the same multivalued function (2.1.11) as in the general minimal model case, while the bosonic correlator (2.2.16) is replaced by

$$
\phi_{\tau}^{(\rho, \rho K)}(u, v, z)=\sum_{|\mu|=S} \prod_{a}\left(\begin{array}{c}
\tau_{a} \\
\mu_{a}
\end{array}\right) \sum_{|\alpha|=s} \prod_{a}\left(\begin{array}{c}
\mu_{a} \\
\alpha_{a}
\end{array}\right) \varphi_{\alpha}^{\rho}(u, z) \varphi_{\mu-\alpha}^{\rho K}(v, z) .
$$

The problem now is that this correlator will not be a meromorphic function any more when we continue back to the true rational values of $J_{a}$ and $K$. In particular in the quasithermal case, i.e., $s=0, S=s^{\prime} K$, involving only the second screening charge operator (2.2.20), we can continue (2.2.14) for non-integer $K$ and $\mu$ such that, $|\mu|=S=s^{\prime} K$, choosing the $n-2$ independent entries of the vector $\mu,|\mu|=S$, and $(n-2)\left(s^{\prime}-1\right)$ of the parameters $A_{i a}$, to be non-negative integers. The rest of the parameters are expressed using the restrictions in (2.2.12), (2.2.13) with $l_{i}=K$ and thus we end up with infinite series, representing a non-meromorphic factor $\varphi_{\mu}^{\rho K}$. More generally we can choose a matrix $A_{0}$ satisfying (2.2.12), (2.2.13), and take the sum in (2.2.14) over all matrices $A$ satisfying these restrictions and differing from $A_{0}$ by integer matrices. Similarly we can generalize the $x_{a}$-expansion (2.2.6) choosing an arbitrary vector $\tau^{(0)}$ satisfying $\left|\tau^{(0)}\right|=2 J_{n}+S$. Then we can sum over $\tau=\left(2 J_{1}, \tau_{2}, \ldots \tau_{n-1}\right)$, satisfying this condition, which differ from $\tau^{(0)}$ by integers. (Recall that $\underline{x}_{1}=\underline{z}_{1}=0$, so that $\tau_{1}=J_{1}-M_{1}=2 J_{1}$. All factorials are replaced by $\Gamma$ - functions.) This gives meaning to the infinite series, which replace the finite sums in (2.2.10).

In general these extensions, leading to different non-meromorphic factors $\varphi_{\mu}^{\rho K}$, are inequivalent.

For example for $n=4$ and $2 J_{2}=2 J_{3}=K, J_{1}=J_{4}$, i.e., $s^{\prime}=1, S=K$, (the case considered in [24]) we can choose $\tau^{(0)}=\left(2 J_{1}, K, 0\right)$ and $\mu=(t, K-t, 0), t=0,1, \ldots$ - then the factor (2.2.16) is simply 3

$$
\phi_{\tau}^{K \rho}(\underline{z})=\sum_{t=0}^{\infty}\left(\begin{array}{c}
2 J_{1} \\
t
\end{array}\right)\left(\begin{array}{c}
K \\
t
\end{array}\right) \frac{K !}{u^{t}(u-\underline{z})^{K-t}} .
$$

Summing furthermore in (2.2.10) over $\tau=\left(2 J_{1}, K-l, l\right), l \in \mathbb{Z}_{+}$we get a power series in $\underline{x}$ for the correlator. This corresponds to $M_{1}=-J_{1}, M_{2}=-K+l, M_{3}=K-l, M_{4}=J_{1}, l \in \mathbb{Z}_{+}$, i. e. to the choice of lowest weight representations of $\operatorname{sl}(2)$ sitting at the first and the second points

\footnotetext{
${ }^{3}$ Here and in what follows we keep for simplicity the notation $a$ ! instead of $\Gamma(a+1)$.
} 
and highest weight representations at the last two points. Another choice, made in [24], is provided by $\tau^{(0)}=\left(2 J_{1}, 0, K\right), \quad \mu=(t, 0, K-t), t=0,1, \ldots$, so that in $(2.2 .23)(u-\underline{z})$ is replaced by $(u-1)$. This choice (taking $\left.\tau=\left(2 J_{1}, K-l, l\right), l \in \mathbb{Z}_{+}\right)$in (2.2.10) corresponds to lowest weight representations at the first and the third points and highest weight representations at the second and the last. The correlator is given by an expression in inverse powers of $\underline{x}$, times $\underline{x}^{2 J}$. Both the choice in [24] and (2.2.23) lead to fusion transformations different from the minimal ones. Namely they give a single contribution $J_{j, j^{\prime}+1 / 2}$ or $J_{j, j^{\prime}-1 / 2}$ respectively, in the product $J_{j, j^{\prime}} \otimes J_{0,1 / 2}$ while both are present in the minimal model fusion rules. The absence of the second contribution is due to non-meromorphic powers like the one in 2.2.23) which "wipes" away the same factor in the function $\Phi^{(0,1 ; \nu)}$.

This example shows that it is rather awkward to model infinite dimensional representations of $g$ (which in general are neither highest nor lowest weight representations) by bosonic powers. So instead of trying to give meaning to the bosonic expressions we shall follow a different way. To understand our motivation it is instructive first to consider the thermal case.

\section{Generalized hypergeometric integrals, the $(x-z)$-expansion and the reduction of the $\mathrm{KZ}-\mathrm{ZF}$ system of equations - the thermal case}

\subsection{Equivalent integral representations of the thermal correlators}

Summarizing the formulae of the previous section, provided by the bosonization construction, we have for the thermal $n$-point correlators (2.2.10)

$$
G_{j}^{(n)}(x, z)=\sum_{|\tau|=s+2 j_{n}} \prod_{a=1}^{n-1}\left(\begin{array}{c}
2 j_{a} \\
\tau_{a}
\end{array}\right) x_{a}^{2 j_{a}-\tau_{a}} \sum_{|\mu|=s} \prod_{a=1}^{n-1}\left(\begin{array}{c}
\tau_{a} \\
\mu_{a}
\end{array}\right) I_{\mu \Gamma}^{(s)}(z) .
$$

Since we will assume that we integrate over a fixed cycle $\Gamma$ we will skip indicating it explicitly. The integral $I_{\mu}$ is defined from the bosonization scheme for $|\mu|=s$. It can be extended to any $\mu, \quad 0 \leq|\mu| \leq s$, assuming that in (2.2.12) $l_{i}=0,1$ for any $i=1,2, \ldots, s$ and summing over all such $\left\{l_{i}\right\}$, consistent with the condition $|l|=|\mu|$, i.e.,

$$
\varphi_{\mu}(u, z)=\frac{(s-|\mu|) !}{s !} \sum_{l} \varphi_{\mu}^{l}(u, z) .
$$

If $|\mu|=s$ the r.h.s. of (3.1.2) contains one term described by $l=\rho$ while for arbitrary $0 \leq|\mu| \leq s$ it reproduces (1.11).

We shall keep the same notation for the corresponding integrals. These generalized hypergeometric integrals satisfy the following relations valid for arbitrary parameters $\nu \neq 0, \infty$ and $\left\{j_{a}, a=1,2, \ldots, n\right\}$, such that $j_{1}+j_{2}+\ldots+j_{n-1}-j_{n}=s, s$ - positive integer:

the linear relation of Aomoto

$$
\sum_{a}\left(2 j_{a}-\mu_{a}\right) I_{\mu+\varepsilon_{a}}^{(s)}=0, \quad\left(\varepsilon_{a}\right)_{b}=\delta_{a b},
$$


the recursion relation

$$
\begin{gathered}
\sum_{a}\left(2 j_{a}-\mu_{a}\right) z_{a} I_{\mu+\varepsilon_{a}}^{(s)}=-c_{|\mu|} I_{\mu}^{(s)}, \\
c_{|\mu|}=\sum_{a=1}^{n} j_{a}-|\mu|+1-\frac{1}{\nu},
\end{gathered}
$$

and the system of differential equations

$$
\begin{aligned}
\frac{1}{\nu} \partial_{a} I_{\mu}^{(s)} & +\sum_{b(\neq a)} \frac{1}{z_{a b}}\left[\mu_{a}\left(2 j_{b}-\mu_{b}\right)\left(I_{\mu}^{(s)}-I_{\mu-\varepsilon_{a}+\varepsilon_{b}}^{(s)}\right)+\mu_{b}\left(2 j_{a}-\mu_{a}\right)\left(I_{\mu}^{(s)}-I_{\mu+\varepsilon_{a}-\varepsilon_{b}}^{(s)}\right)\right] \\
& =\left(2 j_{a}-\mu_{a}\right)(s-|\mu|) I_{\mu+\varepsilon_{a}}^{(s)}, \quad a=1, \ldots, n-1 .
\end{aligned}
$$

In the case under consideration $\nu=1 /(k+2)$ and $0 \leq|\mu| \leq s$. The relations (3.1.3) (for $|\mu|=s$ ) and (3.1.4) were derived in [22]. The equation (3.1.6) is an extension for $|\mu| \leq s$ of the corresponding equation derived in 22] for $|\mu|=s$. The proof of these relations will be reproduced in Sect. 4 as a particular case of a more general computation.

Now we will show that the conformal block (2.2.17) coming from the bosonization can be rewritten in terms of a single hypergeometric integral, namely

$$
\mathcal{G}_{\left(2 j_{1}, \tau_{2}, \ldots, \tau_{n-1}\right)}(\underline{z})=(-1)^{s} I_{\left(0,2 j_{2}-\tau_{2}, \ldots, 2 j_{n-1}-\tau_{n-1}\right)}^{(s)}(\underline{z}) .
$$

We have taken $\tau_{1}=2 j_{1}$ since, using the isospin projective invariance we can always choose $x_{1}=0$, so that in the correlator (2.2.8) only the component $m_{1}=-j_{1}$, i.e., $\tau_{1}=2 j_{1}$, survives.

To prove (3.1.7) we first iterate the Aomoto linear relation (3.1.3) obtaining

$$
I_{\mu}^{(s)}=\frac{(-1)^{\mu_{1}-\sigma_{1}}\left(\mu_{1}-\sigma_{1}\right) !\left(2 j_{1}-\mu_{1}\right) !}{\left(2 j_{1}-\sigma_{1}\right) !} \sum_{\sigma_{2}, \ldots, \sigma_{n-1}} \prod_{a=2}^{n-1}\left(\begin{array}{c}
2 j_{a}-\mu_{a} \\
2 j_{a}-\sigma_{a}
\end{array}\right) I_{\sigma}^{(s)}
$$

where $|\mu|=|\sigma|=s$. Substituting this on the r.h.s. of (2.2.17) with $\tau_{1}=2 j_{1}, \sigma_{1}=0$ we obtain

$$
(-1)^{s} \sum_{\sigma_{2}, \ldots, \sigma_{n-1}} \sum_{\mu_{2}, \ldots, \mu_{n-1}} \prod_{a=2}^{n-1}\left(\begin{array}{c}
2 j_{a}-\mu_{a} \\
2 j_{a}-\sigma_{a}
\end{array}\right)\left(\begin{array}{c}
\tau_{a} \\
\mu_{a}
\end{array}\right)(-1)^{\mu_{a}} I_{\left(0, \sigma_{2}, \ldots, \sigma_{n-1}\right)}^{(s)}(\underline{z})
$$

This can be rewritten as

$$
(-1)^{s} \sum_{\sigma_{2}, \ldots, \sigma_{n-1}} \prod_{a=2}^{n-1} \frac{\left(2 j_{a}-\tau_{a}\right) !}{\sigma_{a} !} \prod_{a=2}^{n-1} \frac{1}{\left(2 j_{a}-\tau_{a}-\sigma_{a}\right) !} I_{\left(0, \sigma_{2}, \ldots, \sigma_{n-1}\right)}^{(s)}(\underline{z})
$$

making use of the ${ }_{2} F_{1}$ hypergeometry summation formula:

$$
\sum_{\mu_{a}}(-1)^{\mu_{a}}\left(\begin{array}{c}
2 j_{a}-\mu_{a} \\
2 j_{a}-\sigma_{a}
\end{array}\right)\left(\begin{array}{c}
\tau_{a} \\
\mu_{a}
\end{array}\right)=\left(\begin{array}{c}
2 j_{a}-\tau_{a} \\
\sigma_{a}
\end{array}\right)
$$

Note that if $A_{a}$ are integers and $\sum A_{a}=0$ then

$$
\prod_{a} \frac{1}{A_{a} !}=\prod_{a} \delta_{A_{a}, 0}
$$


Obviously

$$
\sum_{a=1}^{n-1}\left(2 j_{a}-\tau_{a}-\sigma_{a}\right)=2\left(s+j_{n}\right)-|\tau|-|\sigma|=0
$$

so with the choice $\tau_{1}=2 j_{1}$ and $\sigma_{1}=0$ we have $\sum_{a=2}^{n-1}\left(2 j_{a}-\tau_{a}-\sigma_{a}\right)=0$ and thus

$$
\prod_{a=2}^{n-1} \frac{1}{\left(2 j_{a}-\tau_{a}-\sigma_{a}\right) !}=\prod_{a=2}^{n-1} \delta_{\sigma_{a}, j_{a}+m_{a}}
$$

Therefore we obtain (3.1.7) which, inserted in (2.2.10), (2.2.17) gives

$$
G_{j}^{(n)}(\underline{x}, \underline{z})=(-1)^{s} \sum_{\substack{|\sigma|=s \\
\sigma:}} \prod_{\substack{a=2 \\
\sigma_{1}=0}}^{n-1}\left(\begin{array}{c}
2 j_{a} \\
\sigma_{a}
\end{array}\right) \underline{x}_{a}^{\sigma_{a}} I_{\sigma}^{(s)}(\underline{z})
$$

The representation (3.1.8) corresponds to the one considered in [15]. More precisely, inserting (3.1.8) in (2.2.8) and using the iterated Aomoto linear relation we can rewrite the $x$-expansion for the correlator at infinity (2.2.9) as

$$
U^{(n)}(x, z)=\prod_{1 \leq a<b \leq n-1} z_{a b}^{2 j_{a} j_{b} \nu} G_{j}^{(n)}(x, z)=\sum_{|\alpha|=s} \prod_{a=1}^{n-1}\left(\begin{array}{c}
2 j_{a} \\
\alpha_{a}
\end{array}\right) x_{a}^{\alpha_{a}} \prod_{1 \leq a<b \leq n-1} z_{a b}^{2 j_{a} j_{b} \nu} I_{\alpha}^{(s)}(z)
$$

Our next step will be to derive another equivalent representation for the thermal correlators. As already argued in the introduction the 2- and 3-point functions of the WZNW model reproduce in the limit $x_{a} \rightarrow z_{a}$ their minimal model counterparts. We will show that this is true for arbitrary $n$-point functions. Indeed we have the following $(x-z)$-expanded form of the correlators

$$
G_{j}^{(n)}(x, z)=\sum_{t=0}^{s} \sum_{|\tau|=t} \prod_{a=1}^{n-1}\left(x_{a}-z_{a}\right)^{\tau_{a}} B_{\tau} I_{\tau}^{(s)}(z)
$$

where

$$
B_{\tau}=\frac{(-1)^{t}}{(s-t) !} \prod_{r=0}^{s-t-1} c_{t+r} \prod_{a=1}^{n-1}\left(\begin{array}{c}
2 j_{a} \\
\tau_{a}
\end{array}\right), \quad t=|\tau| .
$$

The crucial element in the derivation of this formula is the recursion relation (3.1.4). Iterating it $s-t$ times we obtain

$$
\sum_{|\sigma|=s} \prod_{a=1}^{n-1} z_{a}^{\sigma_{a}-\tau_{a}}\left(\begin{array}{c}
2 j_{a}-\tau_{a} \\
\sigma_{a}-\tau_{a}
\end{array}\right) I_{\sigma}^{(s)}=(-1)^{s-t} \frac{\prod_{r=0}^{s-t-1} c_{t+r}}{(s-t) !} I_{\tau}^{(s)}
$$

Now expand

$$
x_{a}^{\sigma_{a}}=\sum_{\tau_{a}}\left(\begin{array}{c}
\sigma_{a} \\
\tau_{a}
\end{array}\right)\left(x_{a}-z_{a}\right)^{\tau_{a}} z^{\sigma_{a}-\tau_{a}},
$$

and use the trivial identity for binomial coefficients

$$
\left(\begin{array}{c}
2 j_{a} \\
\sigma_{a}
\end{array}\right)\left(\begin{array}{c}
\sigma_{a} \\
\tau_{a}
\end{array}\right)=\left(\begin{array}{c}
2 j_{a} \\
\tau_{a}
\end{array}\right)\left(\begin{array}{c}
2 j_{a}-\tau_{a} \\
\sigma_{a}-\tau_{a}
\end{array}\right)
$$


to rewrite $G_{j}^{(n)}(x, z)$ in $(3.1 .9)$ as

$$
\sum_{t=0}^{s}(-1)^{t} \sum_{|\tau|=t} \prod_{a=1}^{n-1}\left(\begin{array}{c}
2 j_{a} \\
\tau_{a}
\end{array}\right)\left(x_{a}-z_{a}\right)^{\tau_{a}}\left[\sum_{|\sigma|=s} \prod_{a=1}^{n-1} z_{a}^{\sigma_{a}-\tau_{a}}\left(\begin{array}{c}
2 j_{a}-\tau_{a} \\
\sigma_{a}-\tau_{a}
\end{array}\right) I_{\sigma}^{(s)}\right] .
$$

Thus, applying the iterated recursion relation for the expression in the brackets gives us the formula for the $(x-z)$ - expansion.

The expansion (3.1.10) simplifies when written for the 4-point function in the system $(0,1, \infty)$

$$
G_{j}^{(4)}(\underline{x}, \underline{z})=\sum_{t}(\underline{x}-\underline{z})^{t} C_{t}(\underline{z})=\sum_{t}(\underline{x}-\underline{z})^{t} B_{t} I_{t}^{(s)}(\underline{z})
$$

where for short we have replaced the vector $\tau=(0, t, 0)$ with the number $|\tau|=t$. The Aomoto differential equations (3.1.6) transform to a system of ordinary differential equations for the integrals $I_{t}^{(s)}(\underline{z})$

$$
\begin{gathered}
\left((k+2) \partial_{z}+a_{t t}\right) I_{t}^{(s)}(\underline{z})=\left(2 j_{2}-t\right)(s-t) I_{t+1}^{(s)}(\underline{z})-\frac{t c_{t}}{z(z-1)} I_{t-1}^{(s)}(\underline{z}), \\
a_{t t}=\frac{A_{t}\left(j_{1}+j_{2}\right)}{z}+\frac{A_{t}\left(j_{3}+j_{2}\right)}{z-1}, \quad A_{s}(j)=s(2 j-s+1) \\
t=0,1,2, \ldots, \min \left(s, 2 j_{2}\right) .
\end{gathered}
$$

To show this we use the recursion relation (3.1.4)

$$
2 j_{3} I_{(0, t-1,1)}^{(s)}+z\left(2 j_{2}-t+1\right) I_{(0, t, 0)}^{(s)}+c_{t-1} I_{(0, t-1,0)}^{(s)}=0
$$

and the Aomoto linear relation (3.1.3)

$$
2 j_{3} I_{(0, t-1,1)}^{(s)}+\left(2 j_{2}-t+1\right) I_{(0, t, 0)}^{(s)}+2 j_{1} I_{(1, t-1,0)}^{(s)}=0 .
$$

We solve for $I_{(1, t-1,0)}^{(s)}$ and $I_{(0, t-1,1)}^{(s)}$ and substitute in (3.1.6) which in this case $(\mathrm{n}=4)$ is simply $(\nu=1 /(k+2))$

$$
\begin{aligned}
(k+2) \partial_{z} I_{(0, t, 0)}^{(s)}= & \left(2 j_{2}-t\right)(s-t) I_{(0, t+1,0)}^{(s)} \\
& -2 j_{1} t \frac{1}{z}\left(I_{(0, t, 0)}^{(s)}-I_{(1, t-1,0)}^{(s)}\right)-2 j_{3} t \frac{1}{(z-1)}\left(I_{(0, t, 0)}^{(s)}-I_{(0, t-1,1)}^{(s)}\right) .
\end{aligned}
$$

We get 3.1.17) which is nothing but the KZ equation (1.1) applied to the 4-point function (2.2.8) with $\Omega_{a b}$ given in general by

$$
\Omega_{a b}=-x_{a b}^{2} \frac{\partial}{\partial x_{a}} \frac{\partial}{\partial x_{b}}+2 x_{a b}\left(J_{a} \frac{\partial}{\partial x_{b}}-J_{b} \frac{\partial}{\partial x_{a}}\right)+2 J_{a} J_{b} .
$$

Written in terms of the coefficients $C_{t}(z)=B_{t} I_{t}^{(s)}(z)$ in the $(x-z)$ - expansion of the correlator (3.1.16), the equation 


$$
\left[(k+2) \partial_{z}+a_{t t}\right] C_{t}(\underline{z})+(t+1) c_{t} C_{t+1}(\underline{z})+\frac{(s-t+1)\left(2 j_{2}-t+1\right)}{z(1-z)} C_{t-1}(\underline{z})=0
$$

first appeared in [11]. Here we have found the solution in terms of the chiral integrals $I_{t}^{(s)}(z)$ and the explicit numerical coefficients $B_{t}$. In [11] a somewhat different parametrization of the (twodimensional) integrals is used with $\left\{j_{a}\right\}$ replaced by (see also [14])

$$
\begin{aligned}
& \tilde{\jmath}_{1}=\frac{1}{2}\left(j_{1}+j_{2}-j_{3}-j_{4}+k+1\right), \\
& \tilde{\jmath}_{2}=\frac{1}{2}\left(j_{1}+j_{2}+j_{3}+j_{4}-k-1\right), \\
& \tilde{\jmath}_{3}=\frac{1}{2}\left(j_{2}+j_{3}-j_{1}-j_{4}+k+1\right), \\
& \tilde{\jmath}_{4}=\frac{1}{2}\left(j_{2}+j_{1}-j_{3}-j_{4}+k+1\right) .
\end{aligned}
$$

This transformation relates the numerical coefficients in (3.1.17) and (3.1.23) so that up to an overall constant, which can be computed using the results of [19], we can make the identification

$$
I_{t}^{(s)}\left(\left\{\tilde{\jmath}_{a}\right\} ; z\right)=\text { const }(s-t) ! t !(-1)^{t} C_{t}^{(s)}\left(j_{a} ; z\right) .
$$

It is straightforward, using the relations (3.1.3), (3.1.4), to show the equivalence, for arbitrary $n$, of (3.1.6) and the KZ - ZF system of equations written using (3.1.22) for $\left\{I_{\tau}^{(s)}(\underline{z}),|\tau| \leq s, \tau_{1}=\right.$ $\left.\tau_{n-1}=0\right\}$.

Both the $x$ - and $(x-z)$ - expansions are finite series in agreement with the algebraic equation corresponding to a singular vector of the $A_{1}^{(1)}$-Verma modules. In the thermal case under consideration it is simply a monomial and can be represented by the derivative of order $2 j_{a}+1$ with respect to $x_{a}$ - exactly as in the integer level $\left(k+2=p ; p^{\prime}=1\right)$ case. In the integer level case the second algebraic equation is reflected in the truncation from below of the $(x-z)$ - expansion if $c_{0} \geq 0$, integer [11], [28]. Indeed, examining the numerical coefficients in the expansion (3.1.16), the lower bound in (3.1.16) is found to be $t_{\min }=\max \left(0, c_{0}+1\right)$ for $p^{\prime}=1$. For general $k$ one expects instead a linear relation for the integrals in the $(x-z)$-expansion (see also Section 4.3).

The expansion (3.1.16), and more generally (3.1.10), makes explicit the reduction of the WZNW correlators to the minimal theory ones in the limit $x_{a} \rightarrow z_{a}$. What we have done (for $n=4$ ) was to change the set of integrals entering the $x$-expansion (3.1.8), provided by the bosonization,

$$
\left\{I_{\mu}^{(s)},|\mu|=s\right\}
$$

subject of the linear relation (3.1.3), to an equivalent (for generic isospin values) set

$$
\left\{I_{t}^{(s)}, t=0,1, \ldots, s\right\},
$$

containing the minimal integral $I_{0}$ as one of its members.

This was done exploiting the recursion relation (3.1.4) which can be equivalently rewritten as

$$
\sum_{a=1}^{n-1} z_{a} \partial_{x_{a}} U_{t}(x, z)=U_{t-1}(x, z)
$$


where $U_{t}(x, z), t=0,1, \ldots, s$ is defined by the r.h.s. of (3.1.9) times an overall constant $(-1)^{t} c_{t} ! / c_{s}$ ! and the condition $|\alpha|=s$ replaced by $|\alpha|=t$. Applying (3.1.4) to the correlator $U^{(n)}\left(=U_{s}\right)$, rewritten using (3.1.10) as a $(x-z)$ - expansion in terms of $U_{t}(x-z ; z)$, we obtain

$$
\sum_{a=1}^{n-1} z_{a} \partial_{x_{a}} U^{(n)}(x, z)=\sum_{t=t_{\min }}^{s-1} \frac{1}{(s-t-1) !} U_{t}(x-z ; z)
$$

while

$$
s U^{(n)}(x, z)=\sum_{t=t_{\min }}^{s} \frac{s}{(s-t) !} U_{t}(x-z ; z)
$$

For $t_{\min }=0$ the right hand sides of $(3.1 .28)$ and $(3.1 .29)$ coincide (yielding the Virasoro correlator $\left.U_{0}\right)$ if $x_{a}=z_{a}, a=1, \ldots, n-1$. Thus the condition $U^{(n)}(z, z)=U_{0}(z)$ is equivalent to the requirement that the operator in the l.h.s. of (3.1.28) reduces to the identity operator. This relates the limit $x_{a} \rightarrow z_{a}$ to the standard constraint condition of the quantum hamiltonian reduction [7] since the operator $\Delta\left(X_{1}^{-}\right) \equiv \sum_{a} z_{a} \partial_{x_{a}}$ corresponds to the KM generator $X_{-1}^{+}$applied to the vacuum state created by the field $\Phi^{J_{n}}\left(x_{n}, z_{n}\right)$ at infinity. In the integer level case the vanishing of the $\left(k-2 j_{n}+1\right)^{\text {th }}$ power of $\Delta\left(X_{1}^{-}\right)$applied on $U^{(n)}$ accounts for the algebraic equation mentioned above, responsible for the truncation of the $(x-z)$ - expansion from below. Similarly the Aomoto linear relation (3.1.3) (for $|\mu|-1=s$ ), reexpressed as a relation for $U^{(n)}, \sum \partial_{x_{a}} U^{(n)}=0$, admits an algebraic interpretation 15 .

The relations (3.1.3) and (3.1.4) can be interpreted as coming from relations in twisted cohomology, that is to say, certain forms are exact with respect to a twisted total derivative $\nabla_{\Phi}=$ $\nabla-\nabla(\log \Phi)$. In [22] it was shown that $\varphi_{\mu} d u_{1} \wedge \ldots \wedge d u_{s}$ with $|\mu|=s$ span the symmetric top twisted cohomology $\left[H^{s}\left(X_{s}, \nabla_{\Phi}\right)\right]^{\Sigma}$ and when the Aomoto linear relations are taken into account one gets a basis. Its dimension is $(s+n-3) ! /(s !(n-3) !)$ for generic spins. The two sets (3.1.25) and (3.1.26) (for $n=4)$ correspond to two different bases in cohomology.

Up to now we have considered a fixed cycle $\Gamma$. For non-rational $k+2$ the general solution of the KZ equation is given by a fundamental set of integrals labelled by the cycles in the dual twisted homology group. In the minimal rational case, i.e., $2 j$ restricted in the domain (1.5), we shall restrict ourselves to an "admissible" set of contours, which provides a linearly independent set of integrals. Then given the chiral solution (3.1.10) one can construct the monodromy invariant 2 -dimensional correlations combining the left and right chiral pieces. Let us consider in more detail the case $n=4$. There are (for generic spins) $s+1$ cycles $\Gamma_{p}, p=1,2, \ldots, s+1$, any $\Gamma_{p}$ describing a set of contours. One can choose these sets of contours and the branches of the function $\Phi^{(s)}$ as in 19]. Then $\left\{I_{t ; p}^{(s)}\right\}$ transform with the same fusion matrix as $\left\{I_{0 ; p}^{(s)}\right\}$, times a sign $(-1)^{t}$. This can be seen using the invariance of the system (3.1.17). The sign is compensated in the power series (3.1.16) when $x$ and $z$ simultaneously go to $(1-x)$ and $(1-z)$, so that the full function (3.1.16) transforms with the same minimal theory fusion matrix. Then the construction of the monodromy invariant correlators repeats the one for the minimal models. Furthermore one can construct "mixed" monodromy invariants combining the $(x, z)$ - depending chiral correlators of the WZNW theory with the corresponding $\bar{z}$-dependent minimal conformal blocks.

\footnotetext{
${ }^{4}$ Symmetric with respect to the action of the permutation group $\Sigma_{s}$ on the screening variables $u_{i} i=1, \ldots, s ;$ top refers to $r$-forms with $r=s$
} 


\subsection{Reduction of the thermal KZ system to the BPZ equations}

Let us start with the reduction of the $\mathrm{KZ}-\mathrm{ZF}$ system for the case $n=4$. It is a first order matrix differential equation for the coefficient functions of the $(x-z)$-expansion of the correlators. This matrix differential equation can be reduced to a scalar (BPZ) equation for the correlators of the minimal models - the latter reflecting the decoupling of the Virasoro null vectors.

It is convenient to rewrite the $\mathrm{KZ}-\mathrm{ZF}$ system (3.1.17) as follows

$$
\left(2 j_{2}-t\right)(s-t) I_{t+1}=D_{t+1} I_{t}+\frac{t c_{t-1}}{z(z-1)} I_{t-1}
$$

where

$$
D_{t+1}=\frac{1}{\nu} \partial_{z}+t\left(\frac{2 j_{1}+2 j_{2}+1-t}{z}+\frac{2 j_{2}+2 j_{3}+1-t}{z-1}\right)
$$

Using (3.2.1) one may express recursively $I_{t}$ by the lower ones and finally represent it as a differential operator of order $t$ acting on $I_{0}$ :

$$
\frac{\left(2 j_{2}\right) !}{\left(2 j_{2}-t\right) !} \frac{s !}{s-t) !} I_{t}=\sum_{k=0}^{\left[\frac{t}{2}\right]} \sum_{\left\{i_{k}, \ldots, i_{1}\right\}} D_{t} \ldots \overbrace{D_{i_{k}+1} D_{i_{k}}} D_{i_{k}-1} \ldots \overbrace{D_{i_{1}+1} D_{i_{1}}} \ldots D_{2} D_{1} I_{0}
$$

where the "pairing" is given by

$$
\overbrace{D_{r+1} D_{r}} \equiv\left(2 j_{2}+1-r\right)(s+1-r) \frac{r c_{r-1}}{z(z-1)}
$$

and the second sum in (3.2.3) is over all subsets $\left\{i_{k}, \ldots, i_{1}\right\}$ of $\{t-1, \ldots, 2,1\}$ with $i_{j+1}-i_{j} \geq 2$ and $[t / 2]$ is the integer part of $t / 2$. The $k=0$ contribution in (3.2.3) does not contain any pairings.

In the thermal case, which we are considering now, the $\mathrm{KZ}-\mathrm{ZF}$ matrix equation is finite and we can carry out its reduction immediately. Indeed, consider the relation (3.2.3) - if we set $t=$ $s_{0} \equiv \min \left(2 j_{2}, s\right)$, then the left-hand side vanishes and we get a scalar differential equation for the integral $I_{0}$.

An explicit expression for the Virasoro singular vectors in the thermal case was written down in [29]. Thus via the conformal Ward identities one can immediately write down the BPZ equations. The ordinary differential equation obtained from (3.2.3) is the null vector decoupling equation for the 4-point function when projective invariance is taken into account, as can be checked immediately in the simplest examples. In fact writing the BPZ equation for the 4-point function as an ordinary differential equation, though straightforward, becomes quite cumbersome as the order of the equation grows. In this respect we may say that the BPZ equation written in the form of (3.2.3) is more explicit. An example of the reduction of the $\mathrm{KZ}$ equation has been also discussed in the context of the coset construction in 30].

The above consideration is not restricted to 4-point functions. Dealing with an arbitrary $n$-point function it is more convenient to send only the last point to infinity instead of working with moduli. We shall reduce the set of equations (3.1.6) which was shown to be equivalent (using the linear and recursion relations (3.1.3), (3.1.4)) to the KZ-ZF system. Using (3.1.6) we can recursively express $I_{\mu}$ as some differential operator acting on $I_{0}$. Doing this in the " $a^{\text {th }}$-direction", $a=1, \ldots, n-1$, i.e., expressing recursively $I_{t \varepsilon_{a}}^{(s)}, t=0,1, \ldots$ we obtain that $I_{\left(s_{a}+1\right) \varepsilon_{a}}^{(s)}$ multiplied by a vanishing coefficient is given by a differential operator acting on $I_{0}^{(s)}$. (Here $s_{a}=\min \left(2 j_{a}, s\right)$ and we shall assume for 
simplicity that $2 j_{a}=s_{a}$ for some $a$.) Then the analogue of (3.2.3) in the case of general $n$ (with only $z_{n}$ fixed at infinity) is

$$
\boldsymbol{L}_{t}=\mathcal{N}_{t}\left(j_{a}\right) \boldsymbol{I}_{0}-\sum_{l=1}^{t} \frac{\nu^{l}}{l !}\left(\prod_{i=0}^{l-1}(t-i)\left(2 j_{a}+1-t+i\right)\right)\left(\left(\sum_{\substack{b=1 \\ b \neq a)}}^{n-1} \frac{j_{b}}{z_{a b}}-\partial_{a}\right)^{l} \cdot 1\right) \boldsymbol{I}_{t-l} .
$$

We have set

$$
I_{\mu}=\nu^{|\mu|} \frac{s !}{(s-t) !} \frac{\prod_{a}\left(2 j_{a}\right) !}{\prod_{a}\left(2 j_{a}-\mu_{a}\right) !} \prod_{1 \leq a<b \leq n-1} z_{a b}^{2 j_{a} j_{b} \nu} I_{\mu}
$$

and for short $\mathbb{I}_{t}=\mathbb{I}_{t \varepsilon_{a}}$ while $\mathcal{N}_{t}\left(j_{a}\right)$ is a differential operator of order $t$, e.g., $\mathcal{N}_{1}=\partial_{a}$,

$$
\begin{gathered}
\mathcal{N}_{2}\left(j_{a}\right)=\partial_{a}^{2}-\nu 2 j_{a} \mathcal{L}_{-2}, \\
\mathcal{N}_{3}\left(j_{a}\right)=\partial_{a}^{3}-\nu\left(2 j_{a} \partial_{a} \mathcal{L}_{-2}+\left(2 j_{a}-1\right) 2 \mathcal{L}_{-2} \partial_{a}\right)+\nu^{2} 2 j_{a}\left(2 j_{a}-1\right) 2 \mathcal{L}_{-3}, \quad \text { etc. },
\end{gathered}
$$

with

$$
\mathcal{L}_{-m}=(-1)^{m} \sum_{b \neq a}^{n-1} \frac{1}{z_{a b}^{m-1}}\left(\frac{(m-1) h_{b}}{z_{a b}}+\partial_{b}\right)
$$

being the standard BPZ differential operator realization for the Virasoro generators 25].

For $t=2 j_{a}+1$ the l.h.s. of (3.2.5) and the second term on the r.h.s. vanish, while $\mathcal{N}_{t}\left(j_{a}\right)$ becomes the operator representing the respective Virasoro singular vector. Hence one recovers the $\mathrm{BPZ}$ equation arising from the decoupling of this vector - given by a descendant of the primary field sitting at the point $z_{a}$.

The form (3.2.5) in which we have cast the KZ system of equations makes explicit its connection with the system written in [31], namely, for any $t$ the first term on the r.h.s. of (3.2.5) can be identified with a state in the $\operatorname{sl}(2)$ module built there. Thus the two systems are related by a kind of Drinfeld - Sokolov "gauge transformation". This relation and the derivation of (3.2.5) will be presented in more detail elsewhere.

\section{Rational level and isospins $A_{1}^{(1)}$ model.}

\subsection{Generalized hypergeometric integrals - the "meromorphic" solution.}

We have written basically two different integral representations for the thermal correlations, the $x$ and $(x-z)$-expansions, and we have shown that they are equivalent. The attempts to extend the first expansion, intrinsically related to the bosonization scheme, for general rational isospin values lead, as we have seen in Sect. 2.2, to non-meromorphic modification factor for the integrand of the minimal theory. On the other hand we can try to find directly a solution of the general KZ equation, which is obtained by inserting in (1.1) the isospin $s l_{I}(2, \mathbb{C})$ generators. Assuming that the solution is represented as a power series in $(x-z)$ we get an infinite system which looks like the ZF system for the coefficients $C_{t}$ (with $j_{a}$ replaced by $J_{a}$ ), only now it is not restricted from above. We will now describe solutions with coefficients characterised by a meromorphic modification function.

Let $\mu, \tau$ and $\sigma$ be $(n-1)$-vectors with non-negative integer components. Set

$$
\varphi_{\mu}^{[L]}\left(\left\{u_{i}, z_{a}\right\}\right)=\frac{(L !)^{m}(m L-|\mu|) !}{(m L) !} \sum_{\left\{N_{i a}\right\}} \frac{\prod_{a} \mu_{a} !}{\prod_{i}\left[\left(L-\sum_{a} N_{i a}\right) ! \prod_{a} N_{i a} !\left(u_{i}-z_{a}\right)^{\left.N_{i a}\right]}\right.} .
$$


Here the sums are finite running over $N_{i a}=0,1, \ldots, \mu_{a}, i=1,2, \ldots, m, a=1,2, \ldots, n-1$, subject to the constraints

$$
\sum_{i=1}^{m} N_{i a}=\mu_{a}
$$

It is not difficult to show that for $\mathrm{L}=1$ and $0 \leq|\mu| \leq s$ (4.1.1) reduces to the thermal factor (2.2.14). Next define

$$
\varphi_{\sigma}^{\left(s, s^{\prime}\right)}\left(\left\{u_{i}, v_{i^{\prime}}, z_{a}\right\}\right)=\frac{\left(s^{\prime} K\right) ! s !}{S !} \sum_{\tau} \prod_{a}\left(\begin{array}{c}
\sigma_{a} \\
\tau_{a}
\end{array}\right)\left(\begin{array}{c}
S-|\sigma| \\
s-|\tau|
\end{array}\right) \varphi_{\sigma-\tau}^{[K]}\left(\left\{v_{i^{\prime}}, z_{a}\right\}\right) \varphi_{\tau}^{[1]}\left(\left\{u_{i}, z_{a}\right\}\right) .
$$

Here $K \equiv-k-2, S=s+s^{\prime} K$ and the sum runs over $\left\{\tau_{a}=0,1, \ldots, s, \quad|\tau| \leq s\right\}$. The expression (4.1.3) is the general non-thermal factor. It reduces to the thermal one when $s^{\prime}=0$ or the quasithermal $\varphi^{[K]}$ when $s=0$. The convention will be that $m$ from 4.1.1) is $s$ in the thermal case $L=1$ while in the quasithermal case $L=K$ it should be identified with $s^{\prime}$ and the screening variables are $v_{i^{\prime}}$ instead of $u_{i}$.

The object of our interest are the generalised hypergeometric integrals

$$
I_{\Gamma, \sigma}^{\left(s, s^{\prime}\right)}=\int_{\Gamma} d u_{1} \ldots d u_{s} d v_{1} \ldots d v_{s^{\prime}} \varphi_{\sigma}^{\left(s, s^{\prime}\right)} \Phi_{J}^{\left(s, s^{\prime} ; \nu\right)} .
$$

where we have used the general multivalued integrand 2.1.11) of the minimal correlations and $J$ is an $(n-1)$ - vector $J=\left(J_{a}=j_{a}+j_{a}^{\prime} K\right)$, while $s, s^{\prime}$ are given in (2.1.6). It is assumed that the set of contours $\Gamma$ represents a cycle in the relevant twisted homology group so that no boundary terms survive. Our main result is summarized in the following

Proposition. The integrals (4.1.4) satisfy the following relations:

the linear relations

$$
\sum_{a}\left(2 J_{a}-\sigma_{a}\right) I_{\sigma+\varepsilon_{a}}^{\left(s, s^{\prime}\right)}=0
$$

the recursion relations

$$
\begin{gathered}
\sum_{a}\left(2 J_{a}-\sigma_{a}\right) z_{a} I_{\sigma+\varepsilon_{a}}^{\left(s, s^{\prime}\right)}+c_{|\sigma|} I_{\sigma}^{\left(s, s^{\prime}\right)}=0 \\
c_{|\sigma|}=\sum_{a=1}^{n} J_{a}-|\sigma|-k-1
\end{gathered}
$$

and the differential equations

$$
\begin{aligned}
(k+2) \partial_{a} I_{\sigma}^{\left(s, s^{\prime}\right)} & +\sum_{b(\neq a)} \frac{1}{z_{a b}}\left[\sigma_{a}\left(2 J_{b}-\sigma_{b}\right)\left(I_{\sigma}^{\left(s, s^{\prime}\right)}-I_{\sigma-\varepsilon_{a}+\varepsilon_{b}}^{\left(s, s^{\prime}\right)}\right)+\sigma_{b}\left(2 J_{a}-\sigma_{a}\right)\left(I_{\sigma}^{\left(s, s^{\prime}\right)}-I_{\sigma+\varepsilon_{a}-\varepsilon_{b}}^{\left(s, s^{\prime}\right)}\right]\right. \\
& =(S-|\sigma|)\left(2 J_{a}-\sigma_{a}\right) I_{\sigma+\varepsilon_{a}}^{\left(s, s^{\prime}\right)}
\end{aligned}
$$


This Proposition holds true for arbitrary $k+2 \neq 0$ and any combination of $\left\{J_{a}=j_{a}-j_{a}^{\prime}(k+2)\right\}$ producing, according to (2.1.6), non-negative integers $s, s^{\prime}$. Its proof is rather technical and the whole of Section 5 is devoted to it.

Repeating the argumentation of the previous section one can show that the system of equations (4.1.8) can be rewritten as the (now infinite) KZ - ZF type system. Thus we immediately obtain that the correlation functions $W_{J}^{(n)}\left(\left\{x_{a}, z_{a}, J_{a}\right\}_{a=1}^{n}\right)(\mathrm{cf.}$ (2.2.8)) with

$$
G_{J}^{(n)}(x, z)=\sum_{t=0} \sum_{\tau:|\tau|=t,} \prod_{a=1}^{n-1}\left(x_{a}-z_{a}\right)^{\tau_{a}} B_{\tau} I_{\tau}^{\left(s, s^{\prime}\right)}(z)
$$

where the integrals $I_{\tau}^{\left(s, s^{\prime}\right)}(z)$ are defined in (4.1.4) with the "meromorphic" $\varphi_{\tau}^{\left(s, s^{\prime}\right)}$ from (4.1.3) and

$$
B_{\tau}=\frac{(-1)^{t} \beta_{J}}{(S-t) !} \frac{\left(c_{t}\right) !}{\left(c_{S}\right) !} \prod_{a=1}^{n-1}\left(\begin{array}{c}
2 J_{a} \\
\tau_{a}
\end{array}\right), \quad t=|\tau|,
$$

are solutions of the $\mathrm{KZ}$ equation. They have the same braiding properties as their Virasoro model counterparts which are obtained when we set $x_{a}=z_{a}$. More precisely the limit $x_{a} \rightarrow z_{a}$ exists if $B_{0} \neq 0$. In (4.1.10) $\beta_{J}$ is an overall constant chosen in such a way that all $B_{\tau}$ are finite. It is necessary because the relative constants $B_{\tau} / B_{0}$ may blow up.

In particular, for values of $k$ and $J_{a}$ as in (1.3), (1.4), (1.5) and choosing an admissible ("physical") set of cycles, we get a set of functions which transform in a way consistent with the general minimal models fusion rules, i.e., the multiplicities $N_{J\left(k_{2}, k_{2}^{\prime}\right)}^{J\left(j, j^{\prime}\right)} J_{\left(k_{1}, k_{1}^{\prime}\right)}$ are given by the same factorized expression as the r.h.s. of (2.1.13).

The integrals (4.1.4) can be found directly - at least for the first several values of $|\mu|$ - requiring that the relations (4.1.5), 4.1.6) hold. This fixes the factor (4.1.1) uniquely providing two solutions - one for $L=1$ recovering the thermal integrals and another for $L=K$. Note that the relations (4.1.5), (4.1.6) and hence the functions 4.1.1), corresponding to these two "one-type screening charges" integrals are different (one has a factor $\left(2 j_{a}^{\prime} K-\mu_{a}\right)$ instead of $\left(2 j_{a}-\mu_{a}\right)$ ). Then one checks that the solutions satisfy the differential equation (4.1.8). However it is technically rather difficult to proceed in this way for higher values of $|\mu|$. Instead of that one can try to follow and generalize the argumentation in the thermal case, where the Aomoto relations (3.1.3), (3.1.4), provided the bridge to the $(x-z)$ - expansion. The idea is first to extend for $|\mu| \leq S=s+s^{\prime} K$ the general bosonic expressions (2.2.14), 2.2.22) (assuming that $K$ and $2 J_{a}+1$ are positive integers) and then do the analytic continuation to the rational values of these parameters at a later stage.

Let $E(r, m)$ consist of sets $E=\left\{i_{1}, \ldots, i_{r}\right\}$ with each $i_{k}$ running over $1, \ldots, m$ independently of the rest. Define the $m$-vector $\varepsilon_{E}=\sum_{i \in E} \varepsilon_{i},\left(\varepsilon_{i}\right)_{j}=\delta_{i j}$ thus $\left(\varepsilon_{E}\right)_{j}=\delta_{i_{1} j}+\ldots+\delta_{i_{r} j}$ and $\left|\varepsilon_{E}\right|=r$. Introduce the function

$$
\varphi_{\mu}^{[L]}=\frac{(L !)^{m}}{(m L) !} \sum_{E \in E(r, m)} \varphi_{\mu}^{L \rho-\varepsilon_{E}}, \quad|\mu|=m L-r
$$

where $\rho$ is the $m$-vector $(1,1, \ldots, 1)$ and $\varphi_{\mu}^{l}$ is defined in (2.2.12), (2.2.13), (2.2.14).

For an $m$-vector $l$ with integer entries and such that $|l|=r$ the number of $E \in E(r, m)$ such that $\varepsilon_{E}=l$ is equal to $|l| !\left(\prod_{i} l_{i} !\right)^{-1}$. Hence we can rewrite the above formula as

$$
\frac{(L !)^{m}}{(m L) !} \sum_{l} \frac{(m L-|\mu|) !}{\prod_{i} l_{i} !} \varphi_{\mu}^{L \rho-l}=\frac{(L !)^{m}}{(m L) !} \sum_{A} \frac{(m L-|\mu|) !}{\prod_{i}\left(L-\sum_{a} A_{i a}\right) !} \frac{\prod_{a} \mu_{a} ! \delta_{\mu_{a}, \sum_{i} A_{i a}}}{\prod_{i, a} A_{i a} !\left(u_{i}-z_{a}\right)^{A_{i a}}} .
$$


Keeping $\left\{\mu_{a}\right\}$ as non-negative integers, we can analytically continue the last formula to noninteger $L$, recovering (4.1.1). Indeed, unlike $\left\{l_{i}\right\}$ in the constraint (2.2.12), the parameter $L$ enters (4.1.12) only in the numerical factors, not restricting the summation variables $A_{i a}$ (denoted by $N_{i a}$ in (4.1.1) ). Note that the meromorphic solution (4.1.12) can itself be represented as an $n$-point bosonic correlation with one of the coordinates taken at infinity (see [10]).

The factor (4.1.1) can be rewritten as

$$
\varphi_{\mu}^{[L]}(u, z)=\frac{(m L-t) !}{(m L) !} \sum_{i_{1}, i_{2}, \ldots, i_{t}} C_{i_{1} i_{2} \ldots i_{t}} \frac{1}{u_{i_{1}}-z_{a_{1}}} \ldots \frac{1}{u_{i_{t}}-z_{a_{t}}}
$$

where $\mu=\epsilon_{a_{1}}+\ldots+\epsilon_{a_{t}}, \quad t=|\mu|$. The constant $C_{i_{1} i_{2} \ldots i_{s}}$ is symmetric with respect to all its variables and has a non-vanishing value for any partition $P$ of $t$; e.g., two coinciding indices correspond to a partition $(t=2+1+1+\ldots+1)$, etc. In the thermal case $L=1$ only the terms with non-coinciding $\left\{i_{1}, . ., i_{t}\right\}$ survive, corresponding to the partition $P=(t=1+1+\ldots+1)$ of $t$.

We can think of our meromorphic solution as generated from the minimal integral $I_{0}^{\left(s, s^{\prime}\right)}$ via the KZ - ZF equation. In the non-thermal (or quasithermal) case this is an infinite matrix system thus there is a functional arbitrariness in the solutions. Choosing the minimal model correlators as "initial conditions" for the "evolution in the discrete time $t$ " given by the KZ-ZF system in the form (3.1.23) reproduces our solution, i.e., the infinite set of integrals $\left\{I_{\Gamma, \sigma}^{\left(s, s^{\prime}\right)}\right\}$, serving as coefficients in the expansion (4.1.9).

Note that there are other solutions of the general KZ equation which do not have this property. E.g., a solution exists representing $G^{(4)}(\underline{x}, \underline{z})$ as a power series in the inverse powers of $(\underline{x}-\underline{z})$ times $(\underline{x}-\underline{z})^{2 J_{2}}$. The integrals serving as coefficients in this series are defined using non-meromorphic factors. For example, in the simplest case $s^{\prime}=1, s=0$ these are obtained by formally replacing $t$ with $2 J_{2}-t$. We should also mention that the proof of the relations (4.1.5), (4.1.6) and the equation (4.1.8) formally extends for integrals defined using analytic continuation of the factors (4.1.11) to non-integer $L$ and $\mu_{a}$, with $r$ assumed to be a non-negative integer. Then, as in the thermal case, the "non-meromorphic" analogue of the system (4.1.8) is truncated from above for $|\sigma|=S$, or $\sigma_{a}=2 J_{a}$.

\subsection{Duality transformation and the truncation of the quasithermal $\mathrm{KZ}-\mathrm{ZF}$ system}

In the quasithermal case (i.e., $s=0, S=K s^{\prime}$ ) the KZ-ZF system is an infinite system. For $n=4$, as in the thermal case, we can write down, replacing $j_{a}$ with $J_{a}$, the analogue of (3.2.3) but this time the left-hand side never vanishes and contrary to the thermal case we do not get an equation of BPZ type for $I_{0}$ from the KZ-ZF system alone. Instead (3.2.3) only describes the higher $I_{n}$ in terms of $I_{0}$. On the other hand, on the level of the minimal theory there is apparently no asymmetry between the "thermal" and "quasithermal" cases. The transformation

$$
K \rightarrow 1 / K \quad J \rightarrow J / K=j_{a}^{\prime}+j_{a} / K
$$

which keeps invariant (1.7), amounts to a transposition of the Kac table, i.e., exchanging the rows and columns of the Kac table, or equivalently the "+" and "-" screening charges. Thus we can start with another WZNW theory with "dual" level and isospin values, whose correlations reduce to the same minimal correlations. In the quasithermal case under consideration the dual set is finite. Comparing the two KZ - ZF systems of equations we can express any of the integrals $\left\{I_{t}^{\left(0, s^{\prime}\right)}\right\}$ in 
the infinite set in terms of this finite basis. This implies additional relations for the quasithermal integrals, namely any of them for $t>s^{\prime}$ will be expressed as a linear combination (with coefficients that in general are rational functions of $z$ ) of the subset $\left\{I_{t}^{\left(0, s^{\prime}\right)}\right\}$ with $t=0,1, \ldots, s^{\prime}$. In this way the equation (3.2.3) effectively truncates and can be furthermore reduced, producing again a BPZ type equation for the DF integrals. We shall present an explicit algorithm to obtain the additional relations for the quasithermal integrals.

If $\mathrm{F}$ is a matrix, denote by $\mathrm{F}^{(k)}$ the matrix consisting of the $k$-th diagonal, i.e., $\mathrm{F}_{i j}^{(k)}=\delta_{j, i+k} \mathrm{~F}_{i, i+k}$. For short denote $\mathrm{F}_{i}^{(k)}=\mathrm{F}_{i, i+k}^{(k)}$. The KZ-ZF system of coupled first-order in $z$ differential equations involves at most three $t$ 's , i.e., $I_{t}, I_{t \pm 1}$. It can be written as the following first-order matrix differential equation for the column vector $\mathrm{I}^{\left(s, s^{\prime}\right)}=\left(I_{t}^{\left(s, s^{\prime}\right)}\right)$ :

$$
(\partial \mathbb{1}+\mathrm{F}) \mathrm{I}^{\left(s, s^{\prime}\right)}=0,
$$

where $\mathbb{1}$ is the unit matrix, $\partial=\partial / \partial z$ and $\mathrm{F}\left(=\mathrm{F}(J, K, S), S=s+s^{\prime} K\right)$ is a tridiagonal matrix with

$$
\begin{aligned}
\mathrm{F}_{t}^{(1)}(z)=\mathrm{F}_{t t+1} & =\frac{1}{K}\left(2 J_{2}-t\right)(S-t) \\
\mathrm{F}_{t}^{(0)}(z)=\mathrm{F}_{t t} & =\frac{-t}{K}\left[\frac{2 J_{1}+2 J_{2}+1-t}{z}+\frac{2 J_{2}+2 J_{3}+1-t}{z-1}\right] \\
\mathrm{F}_{t}^{(-1)}(z)=\mathrm{F}_{t t-1} & =\frac{-t}{K} \frac{c_{t-1}}{z(z-1)}
\end{aligned}
$$

and all other diagonals zero. We recall

$$
c_{t}=\sum_{a=0}^{4} J_{a}-t+1+K .
$$

For short let us denote by $I_{t}=I_{t}^{(s, 0)}(J, K)$ and $\mathrm{F}=\mathrm{F}(J, K, S) \quad(S=s)$ the thermal ones while their quasithermal dual counterparts we will denote by $\hat{I}_{t}=I_{t}^{(0, s)}(\hat{J}, \hat{K})$ and $\hat{\mathrm{F}}=\mathrm{F}(\hat{J}, \hat{K}, \hat{S})$ where $\hat{K}=1 / K, \hat{J}=J / K$ and $\hat{S}=S / K=\hat{K} s$. Obviously

$$
\hat{I}_{0}=I_{0}
$$

We want to determine a lower triangular matrix $\mathrm{E}$, i.e. $\mathrm{E}=\mathrm{E}^{(0)}+\mathrm{E}^{(-1)}+\mathrm{E}^{(-2)}+\ldots$, (a DrinfeldSokolov [6] "gauge transformation") transforming $\left(I_{t}\right)$ into $\left(\hat{I}_{t}\right)$ :

$$
\hat{I}_{t}=\mathrm{E}_{t}^{(0)} I_{t}+\mathrm{E}_{t}^{(-1)} I_{t-1}+\mathrm{E}_{t}^{(-2)} I_{t-2}+\ldots+\mathrm{E}_{t}^{(-t)} I_{0}
$$

and such that

$$
\mathrm{E}(\partial+\mathrm{F})=(\partial+\hat{\mathrm{F}}) \mathrm{E},
$$

i.e., the effectively truncated equation is equivalent to its dual. An algorithm for the recursive determination of the entries of the matrix $\mathrm{E}$ is given in the Appendix.

Let us illustrate in the example $s=1$ the linear dependence we were talking about, i.e., of the quasithermal integrals $\hat{I}_{t}=I_{t}^{(0,1)}(\hat{J}, \hat{K})$ only $\hat{I}_{0}$ and $\hat{I}_{1}$ are independent. We have that $s=1$ implies 
$\mathrm{E}_{2}^{(0)}=0$ (see $(\mathrm{A.3})$ ), reflecting the finiteness of the thermal system. Thus $\hat{I}_{2}=\mathrm{E}_{2}^{(-1)} I_{1}+\mathrm{E}_{2}^{(-2)} I_{0}$. We have $\hat{I}_{0}=I_{0}$. Also $\hat{I}_{1}=I_{1}$ because $\mathrm{E}_{1}^{(0)}=1$ and $\mathrm{E}_{1}^{(-1)}=0$ (see above). Therefore

$$
\hat{I}_{2}=\mathrm{E}_{2}^{(-1)} \hat{I}_{1}+\mathrm{E}_{2}^{(-2)} \hat{I}_{0}
$$

with the coefficients given in (A.4) and (A.5) with $s=1$.

This furthermore extends to $C_{t}(z)=B_{t} I_{t}^{(0, t)}(z)$ for arbitrary $t$, i.e.,

$$
\begin{aligned}
& C_{t+2}(z)=a_{t+1}\left(\frac{1}{z}+\frac{1}{z-1}\right) C_{t+1}(z)+b_{t} \frac{1}{z(z-1)} C_{t}(z) \\
& a_{t+1}=\frac{(2 K-t)}{(t+2)(3 K-t)}, \quad b_{t}=\frac{(K-t)^{2}(K-1-t)}{(t-3 K)(t+1)(t+2)},
\end{aligned}
$$

where for simplicity we have chosen all $2 J_{a}=K, a=1, \ldots, 4$. This simple relation can also be derived directly using the explicit expressions for the integrals $I_{t}^{(0,1)}$ and it is equivalent to a standard relation for the solutions of the hypergeometric equation.

The numerical coefficients in the recurrence linear relation (4.2.11) are finite in the limit $t \rightarrow \infty$. This implies that for any fixed $z, z \neq 0,1$ there is a finite region for $|x-z|$, namely

$$
\frac{|x-z|}{|z|}<1 ; \quad \frac{|x-z|}{|1-z|}<1
$$

in which the $(x-z)$ - power series, representing the correlation in this simple example, is absolutely convergent.

We stress that the duality does not mean that there is a direct relation between the correlators of the two theories. One is described by finite and the other by infinite-dimensional representations of the algebra $s l(2, \mathscr{C})$. The quasithermal correlators can be written as a finite $(x-z)$ - expansion, but with coefficients depending on $x$ and $z$.

The analysis above can be extended to arbitrary $n$-point functions in a way analogous to what was done at the end of Section 3.2. In particular we can compare the thermal system written in the form (3.2.5) with its dual quasithermal analogue. Then the vanishing of $\mathcal{N}_{2 j_{a}+1}\left(j_{a}\right)$ (i.e., the thermal BPZ equation) is equivalent to a linear relation involving the quasithermal integrals $I_{\mu}$ with $|\mu| \leq \min \left(2 j_{a}+1, s\right)$.

\subsection{Truncation in the general non-thermal case}

The duality of the thermal and the quasithermal theories established in the previous subsection has a natural cohomological interpretation. The generalized hypergeometric integrals which appear as coefficients in the $(x-z)$ - expansion are integrals of top twisted differential forms. Since the cohomology is finite-dimensional, only a finite number of these integrals are linearly independent (over the rational functions of $z$ ). Indeed since the "twisting" function $\Phi_{\hat{J}}^{(0, s ; \hat{\nu})}=\Phi_{J}^{(s, 0 ; \nu)}(\hat{\nu}=$ $1 / \nu=-K)$ is identical in both theories and the factor $\varphi_{t}^{[K]}$ 4.1.1) for any $t$ is a rational function, the corresponding form $\varphi_{t}^{[K]} d u_{1} \wedge \ldots \wedge d u_{s}$ can be expressed (up to an exact form $\nabla_{\Phi} f$ ) in terms of a standard basis in the symmetric cohomology $\left[H^{s}\left(X_{s}, \nabla_{\Phi}\right)\right]^{\Sigma}, \Phi=\Phi_{J}^{(s, 0 ; \nu)}$. In the case under consideration such a standard basis is provided for generic isospins by the thermal (symmetrized) set (1.11), with $|\mu|=s, \mu_{1}=0$. 
Let us now turn to the general non-thermal case. The situation here is more subtle. Indeed both the initial and the dual theories are described by infinite-dimensional sets of integrals and we cannot truncate the KZ-ZF system exploiting this duality. On the other hand, since our solution is again described by a meromorphic function we can apply the cohomological argumentation to show that there is a finite subset of basic integrals, so that again the system can in principle be truncated. The general twisted cohomology group $H^{m}\left(X_{m}, \nabla_{\Phi}\right)$ [22], associated to a multivalued function of the type in (1.10) with general (nonsymmetric) values of the exponents $\left\{\lambda_{i a}, \lambda_{i j}, i, j=1 \ldots, m ; a=\right.$ $1,2, \ldots, n-1\}$, is spanned by a finite set of logarithmic differential $m$-forms. The reader can find in [22] a linear relation for these $m$-forms generalizing the symmetric (thermal) linear relation (3.1.3) as well as a generalization of the differential system of equations (3.1.6) (a Gauss - Manin system) for the corresponding integrals. The rank of this cohomology computed for generic values of the exponents is $(m+n-3) ! /(n-3)$ ! .

The non-thermal integrals with $m=s+s^{\prime}$ and a set of exponents given in (2.1.12) can in principle be described by this general twisted cohomology. Each of the factors in (4.1.3) corresponds to a rational form and hence can be expressed in terms of the finite basis in [22]. That would imply the truncation of the KZ-ZF equations. On the other hand the multivalued function $\Phi_{J}^{\left(s, s^{\prime} ; \nu\right)}$ in the nonthermal integrals actually provides a very specific example of the general "twisting" factor. Indeed, it is given by an expression that is a product of two thermal (depending on $u_{i}$ and $v_{i^{\prime}}$ respectively) factors that are "coupled" by integer exponents $\lambda_{i, i^{\prime}}=-2$. That can lead to the effective reduction of the number of the basic integrals (at least on an "admissible" subset of contours) to the product of the ranks of the corresponding symmetric cohomologies. (Here, for simplicity, we assume that the spins $\left\{j_{a}, j_{a}^{\prime}\right\}$ are small enough in comparison with $p, p^{\prime}$.)

Let us illustrate this by a simple example for $n=4$ and $m=s+s^{\prime}=2$. We can integrate any $\Phi^{(1,1 ; \nu)}$ - twisted 2 -form $\varphi(u, v) d u \wedge d v$ with respect to one of the variables, say $u$, over a closed contour around the second variable $v$. This defines an operator $\mathcal{J}$ from the cohomology $H^{2}\left(X_{2}, \nabla_{\Phi}\right)$ (of rank six) to the cohomology $H^{1}\left(X_{1}, \nabla_{\tilde{\Phi}}\right.$ ) (of rank two), where $\Phi=\Phi_{J}^{(1,1)}(u, v)$ ), $\left.\tilde{\Phi}=\Phi_{\tilde{J}}^{(1)}(u)\right), \tilde{J}=J(K+1) / K$. The range of $\mathcal{J}$ is 2 -dimensional hence its kernel is 4 -dimensional. For example, if $\varphi_{\mu}=\varphi_{0}$, the integral over $u$ above reduces to the unique linear relation (3.1.3) for the 2 - dimensional cohomology. One can check inductively, starting from $\varphi_{0}$ (i.e., from the minimal integral) and using the $\mathrm{KZ}$ equation, that any of the meromorphic forms in the infinite set of integrals $\left\{I_{(0, t, 0)}, t=0,1,2 \ldots\right\}$ is in the kernel of this operator.

Although this interpretation of the non-thermal theory explains in principle the truncation of the KZ-ZF system, it is not easy to perform it explicitly. Indeed (following the strategy in the quasithermal case), in order to relate the infinite $\mathrm{KZ}-\mathrm{ZF}$ system and the finite system in [22] written for different sets of integrals - we first have to rewrite the latter in another finite basis, which includes the minimal integrals $I_{0}^{\left(s, s^{\prime}\right)}$.

On the other hand there is another more direct mechanism for finding explicitly the relevant additional relations for the coefficients in the $(x-z)$-expansion. Namely we can take into account the algebraic equations for the primary field correlation functions, corresponding to the singular vectors of the KM Verma modules [32].

We recall that in the integrable case $k+2=p$ there are two generating equations, which are differential equations in the functional $x$-realization. They correspond to monomials of the $A_{1}^{(1)}$ generators $f_{1}=X_{0}^{-}$and $f_{0}=X_{-1}^{+}$acting on the vacuum, of degrees $2 j+1$ and $p-2 j-1$ respectively. In the case of rational $k$ the structure of the reducible $A_{1}^{(1)}$ Verma modules $V\left(k, J_{j, j^{\prime}}\right)$ is essentially of the same type. However the corresponding singular vectors labelled by $J_{-j-1, j^{\prime}}$ and 
$J_{p-j-1, j^{\prime}}$ ( the first two in an infinite sequence) are realized in general by homogeneous polynomials $\mathcal{P}_{t_{1}, t_{0}}\left(f_{1}, f_{0}\right) v_{0}$ of both $f_{1}$ and $f_{0}$, of degrees

$$
t_{1}=(2 j+1)\left(2 j^{\prime}+1\right), \quad t_{0}=(2 j+1) 2 j^{\prime},
$$

and

$$
t_{1}=(p-2 j-1)\left(p^{\prime}-2 j^{\prime}-1\right), t_{0}=(p-2 j-1)\left(p^{\prime}-2 j^{\prime}\right),
$$

respectively. The first of these vectors is also present for arbitrary $k+2 \neq 0$ and isospin $J_{j, j^{\prime}}$ as in (1.3).

The general formulae for these polynomials [33] look rather unexplicit. For an illustration we shall use a simple explicit formula presented in [34] which holds for the cases $t_{1}=2 t_{0}$ or $t_{0}=2 t_{1}$ respectively. Namely the singular vector corresponding to (4.3.1), i.e., when $2 j^{\prime}+1=2, j$ is arbitrary, reads

$$
v_{s}=\sum_{s=0}^{2 m}(-1)^{s}\left(\begin{array}{c}
2 m \\
s
\end{array}\right) \frac{m_{1}}{m_{1}-s}\left(f_{1}\right)^{2 m-s}\left(f_{0}\right)^{m}\left(f_{1}\right)^{s} v_{0}
$$

where $m_{1}=2 J+1, m=m_{1}-K(=2 j+1)$.

For $t_{0}=2 t_{1}$ the same formula can be used for the case in 4.3.2 (i.e., $2 j^{\prime}+1=p^{\prime}-1$, $m=p-2 j-1$ - arbitrary), interchanging in (4.3.3) $\left(m_{1}, f_{1}\right)$ and $\left(m_{0}, f_{0}\right)$, where $m_{0}=k+2-m_{1}$. Our convention for the $A_{1}^{(1)}$ commutation relations is

$$
\left[X_{n}^{\alpha}, X_{m}^{\beta}\right]=f_{\gamma}^{\alpha \beta} X_{n+m}^{\gamma}+n q^{\alpha \beta} \delta_{n+m, 0} k,
$$

where

$$
f_{ \pm}^{0 \pm}= \pm 2, f_{0}^{+-}=1, \quad q^{00}=2, q^{+-}=1=q^{-+} .
$$

The decoupling of the null vector translates via the Ward identities to an equation for the $n$-point functions. In the simplest case $2 j^{\prime}=1, j=0$, i.e., $2 J+1=K+1$, and hence $m=1$; when applied to the 4-point function (for $2 j_{n}^{\prime}=1$ ) the equation corresponding to (4.3.1) leads to a relation for the quasithermal integrals $I_{t}, I_{t+1}, I_{t+2}$ for any $t \geq 0$. It coincides exactly with the relation (4.2.11) found above using the duality arguments and we recall that the same linear relation was shown to hold using the explicit expressions for the set of integrals solving the KZ equation. One can expect that this remains true in general, i.e., the relations resulting from duality are equivalent in the quasithermal case to the algebraic equations corresponding to (4.3.1) when $2 j+1=1$.

The next example covered by the formulae (4.3.3), (4.3.1) is the simplest non-thermal example $2 j=1=2 j^{\prime}$, i.e., $m_{1}=2 J+1=2+K, m=2 j+1=2$. In this case one expects a relation involving 5 consequent integrals in the $(x-z)$ - expansion, in particular a relation expressing $I_{4}$ in terms of $I_{t}, t=0,1,2,3$. In other words we would get a system of rank four, i.e., a $4^{\text {th }}$ order equation for the minimal DF correlation function.

Thus in general one can use one of the algebraic equations (that corresponding to (4.3.1) ) in order to select the identities which lead to the truncation of the KZ-ZF system from above. Yet it is rather difficult technically to do this explicitly, since these algebraic equations - being actually differential equations with respect to the variables $x_{a}$ - are very complicated. 


\section{Relations in cohomology and differential equations}

In this section we will derive cohomological relations (the linear and recursion relations) and differential equations for the integrals under consideration.

\subsection{Twisted cohomology}

We recall our convention: $a, b$ are indices (labelling points $z_{a}, z_{b}$ ) which run from 1 to $n-1 ; i, j$ (or $i^{\prime}, j^{\prime}$ ) run from 1 to $s$ (or $s^{\prime}$ ) and label the insertion points $u_{i}, u_{j}$ of type 1 (or type 2) screening operators. When we derive simultaneously the thermal and quasithermal relations we will assume $1 \leq i, j \leq m$, thus $m=s$ in the thermal and $m=s^{\prime}$ in the quasithermal case. Also $\sum_{i}$ will mean summation from 1 to $m$ while $\sum_{a}$ will mean summation from 1 to $(n-1)$. The same will apply to products. Also $\partial_{i}$ and $\partial_{a}$ will be partial derivatives with respect to $u_{i}$ and $z_{a}$, respectively. Denote by $\varepsilon_{i}$ the $m$ vector with $\left(\varepsilon_{i}\right)_{j}=\delta_{i j}$ while $\varepsilon_{a}$ is an $(n-1)$ vector with $\left(\varepsilon_{a}\right)_{b}=\delta_{a b}$. Let $l=\left(l_{i}\right)$ and $\mu=\left(\mu_{a}\right)$ be $m$ and $(n-1)$ - vectors, respectively.

It is convenient to introduce some notation. Define

$$
\psi^{\mu}=\prod_{a}\left(\sum_{i} \frac{1}{\left(u_{i}-z_{a}\right)}\right)^{\mu_{a}}
$$

which can be written also as

$$
\psi^{\mu}=\sum_{A \in \mathcal{A}_{\mu}} \frac{\mu !}{A !(u-z)^{A}}
$$

where the sum is over rectangular matrices $A=\left(A_{i a}\right)$ from the set $\mathcal{A}_{\mu}=\left\{A: \sum_{i} A_{i a}=\mu_{a}, \quad a=\right.$ $1, \ldots, n-1\}$ and we have adopted for short a multi-index notation $\mu !=\prod_{a} \mu_{a} !, A !(u-z)^{A}=$ $\prod_{a} \prod_{i} A_{i a} !\left(u_{i}-z_{a}\right)^{A_{i a}}$. For the monomials in (5.1.2) define $\operatorname{deg}_{i} \prod_{i, a}\left(u_{i}-z_{a}\right)^{-A_{i a}}=\sum_{a} A_{i a}=l_{i}$ and let $P_{i}^{l_{i}}$ project on functions having degree $l_{i}$ in $u_{i}$. For an $m$ vector $l=\left(l_{i}\right)$ define $P^{l}=\prod_{i} P_{i}^{l_{i}}$. Then we have

$$
\varphi_{\mu}^{l} \equiv P^{l} \psi^{\mu}=\sum_{A \in \mathcal{A}_{\mu}^{l}} \frac{\mu !}{A !(u-z)^{A}}
$$

where $\mathcal{A}_{\mu}^{l}=\left\{A \in \mathcal{A}_{\mu}: \sum_{a} A_{i a}=l_{i}, \quad i=1, \ldots, m\right\}$. Notice that $\varphi_{\mu}^{l} \equiv P^{l} \psi^{\mu}$ is different from zero only if

$$
|l|=\sum_{i} l_{i}=\sum_{a} \mu_{a}=|\mu| .
$$

Obviously these projectors satisfy

$$
\partial_{i} P^{l-\varepsilon_{i}}=P^{l} \partial_{i}, \quad \frac{1}{\left(u_{i}-z_{a}\right)} P^{l-\varepsilon_{i}}=P^{l} \frac{1}{\left(u_{i}-z_{a}\right)} .
$$

In 4.1.11) we have introduced $\varphi_{\mu}^{[L]}$. We will also need

$$
\varphi_{\mu}^{[L], i}=\frac{(L !)^{m}}{(m L) !} \sum_{E \in I(r-1, m)} \varphi_{\mu}^{L \rho-\varepsilon_{E}-\varepsilon_{i}}, \quad r=L m-|\mu| .
$$

We will use $\varphi_{\mu}^{[L]}$ to denote both the function and the $m$-form $\varphi_{\mu}^{[L]} d u_{1} \wedge \ldots \wedge d u_{m}$ because either our statements will apply for functions and forms or it will be clear from the context what is assumed. 
The same applies for $\varphi_{\mu}^{[L], i}$. All these forms are considered as representing classes in the twisted cohomology with coefficients in the local system

$$
\Phi^{[L]}=\prod_{i<j} u_{i j}^{2 \nu} \prod_{i, a}\left(u_{i}-z_{a}\right)^{2 \lambda_{a}}
$$

That is to say, in place of the "untwisted" exterior derivative $d=\sum_{i} \partial_{i} d u_{i}$ we have the covariant derivative

$$
D^{[L]}=\sum_{i} D_{i}^{[L]} d u_{i}, \quad D_{i}^{[L]}=\frac{1}{L}\left(\partial_{i}+\partial_{i} \log \Phi^{[L]}\right) .
$$

Let us emphasize that this local system is invariant under permutation of the $u$ 's. P Our notation is designed to treat simultaneously the thermal and quasithermal cases. Integrating the (quasi)thermal forms $\varphi_{\mu}^{[L]}$ one obtains the (quasi)thermal integrals

$$
I_{\mu}^{[L]}(z)=\int \Phi^{[L]}(u, z) \varphi_{\mu}^{[L]}(u, z) .
$$

In the thermal case we have

$$
L=1, \quad \nu=\frac{1}{k+2}, \quad \lambda_{a}=\frac{-J_{a}}{k+2}, \quad m=s
$$

and obviously $\Phi^{[1]} \equiv \Phi_{J}^{(\nu)}(u, z) \equiv \Phi_{J}^{(s, 0 ; \nu)}$. In the quasithermal case

$$
L=K \equiv-(k+2), \quad \nu=k+2, \quad \lambda_{a}=J_{a}, \quad m=s^{\prime}
$$

and the screening variables in this case are $v_{i^{\prime}}$ instead of $u_{i}$ with $i^{\prime}=1, \ldots, s^{\prime}$. The identification $\Phi^{[K]} \equiv \Phi_{-J / \nu}^{(\nu)}(v, z) \equiv \Phi_{J}^{\left(0, s^{\prime} ; \nu^{-1}\right)}$ is again obvious. The flat connection coming from the local system can be written in a unified way for the thermal and quasithermal cases

$$
\frac{1}{L} \partial_{i} \log \Phi^{[L]}=\frac{1}{K}\left(\sum_{a} \frac{2 J_{a}}{\left(u_{i}-z_{a}\right)}-2 L \sum_{j(\neq i)} \frac{1}{\left(u_{i}-u_{j}\right)}\right)
$$

The non-thermal local system was also introduced: $\Phi_{J}^{\left(s, s^{\prime} ; \nu\right)}=\Phi^{[1]} \Phi^{[K]} \prod_{i, i^{\prime}}\left(u_{i}-v_{i^{\prime}}\right)^{-2}$. The coboundary operator is

$$
D=D^{[1]}+D^{[K]}
$$

with $D^{[1]}=\sum_{i} D_{i}^{[1]} d u_{i}$ where

$$
D_{i}^{[1]}=\left(\partial_{i}+\partial_{i} \log \Phi_{J}^{\left(s, s^{\prime} ; \nu\right)}\right)=\partial_{i}+\partial_{i} \log \Phi^{[1]}-2 \sum_{i^{\prime}}\left(u_{i}-v_{i^{\prime}}\right)^{-1}
$$

and $D^{[K]}=\sum_{i^{\prime}} D_{i^{\prime}}^{[K]} d v_{i^{\prime}}$ where

$$
D_{i^{\prime}}^{[K]}=\frac{1}{K}\left(\partial_{i^{\prime}}+\partial_{i^{\prime}} \log \Phi_{J}^{\left(s, s^{\prime} ; \nu\right)}\right)=\frac{1}{K}\left(\partial_{i^{\prime}}+\partial_{i^{\prime}} \log \Phi^{[K]}+2 \sum_{i}\left(u_{i}-v_{i^{\prime}}\right)^{-1}\right)
$$

\footnotetext{
${ }^{5}$ The permutation symmetry is made explicit in the forms (4.1.11) by the averaging over $E$.
} 
In the next subsection we will derive relations which are equivalent to the following cohomological relation $\left(\prod_{j(\neq i)} d u_{j}\right.$ is shorthand for $\left.(-1)^{i+1} d u_{1} \wedge \ldots d u_{i-1} \wedge d u_{i+1} \ldots d u_{m}\right)$

$$
D^{[L]} \sum_{i} \varphi_{\mu}^{[L], i} \prod_{j(\neq i)} d u_{j} \simeq 0
$$

in the (quasi)thermal case and to

$$
D\left(\sum_{i} \varphi_{\sigma}^{\left(s, s^{\prime}\right), i} \prod_{j(\neq i)} d u_{j} \prod_{j^{\prime}} d v_{j^{\prime}}+\sum_{i^{\prime}} \varphi_{\sigma}^{\left(s, s^{\prime}\right), i^{\prime}} \prod_{j} d u_{j} \prod_{j^{\prime}\left(\neq i^{\prime}\right)} d v_{j^{\prime}}\right) \simeq 0
$$

in the general case. The form $\varphi_{\sigma}^{\left(s, s^{\prime}\right)}$ was introduced in (4.1.3) while $\varphi_{\sigma}^{\left(s, s^{\prime}\right), i}$ (or $\varphi_{\sigma}^{\left(s, s^{\prime}\right), i^{\prime}}$ ) are obtained if in (4.1.3) one replaces $\varphi_{\tau}^{[1]}$ by $\varphi_{\tau}^{[1], i}$ (or $\varphi_{\sigma-\tau}^{[K]}$ by $\varphi_{\sigma-\tau}^{[K], i^{\prime}}$ ). Here with $\simeq$ we denote equivalence in cohomology 10 The recursion relations which we will derive in subsection 3 are equivalent to

$$
D^{[L]} \sum_{i} u_{i} \varphi_{\mu}^{[L], i} \prod_{j(\neq i)} d u_{j} \simeq 0
$$

for the (quasi)thermal case and

$$
D\left(\sum_{i} u_{i} \varphi_{\sigma}^{\left(s, s^{\prime}\right), i} \prod_{j(\neq i)} d u_{j} \prod_{j^{\prime}} d v_{j^{\prime}}+\sum_{i^{\prime}} v_{i^{\prime}} \varphi_{\sigma}^{\left(s, s^{\prime}\right), i^{\prime}} \prod_{j} d u_{j} \prod_{j^{\prime}\left(\neq i^{\prime}\right)} d v_{j^{\prime}}\right) \simeq 0
$$

in the general case. Since the hypergeometric integrals are assumed to be over closed (in the twisted homology) contours (cycles), the cohomological identities for the forms turn into ordinary identities for the integrals.

The derivations in the next subsections are very technical but basically they will involve the above cohomological identities, partial fraction expansions and the following formulae:

$$
\sum_{a} \mu_{a} P^{l} \frac{1}{\left(u_{i}-z_{a}\right)} \psi^{\mu-\varepsilon_{a}}=l_{i} P^{l} \psi^{\mu}
$$

or

$$
\sum_{a} \frac{\mu_{a}}{\left(u_{i}-z_{a}\right)} \varphi_{\mu-\varepsilon_{a}}^{[L], i}=L \varphi_{\mu}^{[L]}-(L m-|\mu|) \varphi_{\mu}^{[L], i} .
$$

To prove the first one, notice that the l.h.s. can be written as

$$
\sum_{a} \sum_{A \in \mathcal{A}_{\mu}^{l}} A_{i a} \frac{\mu !}{A !(u-z)^{A}}
$$

Taking first the sum over $a$ we obtain the r.h.s. of (5.1.19). To prove the second formula we should average over $l$ in (5.1.19) and notice that

$$
\sum_{E \in E(r, m)}\left(\varepsilon_{E}\right)_{i} \varphi_{\mu}^{L \rho-\varepsilon_{E}}=r \varphi_{\mu}^{[L], i}
$$

\footnotetext{
${ }^{6}$ All our cohomological relations are for the top forms, i.e., $m$-forms in the (quasi)thermal case and $\left(s+s^{\prime}\right)$-forms in the non-thermal case.
} 


\subsection{Linear relations}

The (quasi)thermal linear relation is

$$
\sum_{a}\left(2 J_{a}-\mu_{a}\right) \varphi_{\mu+\varepsilon_{a}}^{[L]} \simeq 0
$$

or, after integrating,

$$
\sum_{a}\left(2 J_{a}-\mu_{a}\right) I_{\mu+\varepsilon_{a}}^{[L]}=0
$$

As we pointed out, these relations follow from the cohomological identity (5.1.15). In order to prove (5.2.1) we will establish the following identity:

$$
\sum_{i} \partial_{i} \varphi_{\mu}^{[L], i}=-\sum_{a} \mu_{a} \varphi_{\mu+\varepsilon_{a}}^{[L]}+2 L \sum_{i \neq j} \frac{1}{\left(u_{i}-u_{j}\right)} \varphi_{\mu}^{[L], i}
$$

In the quasithermal case the relation (5.2.1) follows immediately from (5.1.15) and (5.2.3) (recall the flat connection given in (5.1.11)). In the thermal case notice that the l.h.s. of (5.2.3) is zero

(becase $\varphi_{\mu}^{[1], i}$ does not depend on $u_{i}$ ). Eliminating the term with $\sum_{i \neq j} u_{i j}{ }^{-1} \ldots$ from (5.2.3) and (5.1.11) we obtain (5.2.1).

Now we will derive (5.2.3). First let us show

$$
\sum_{i} \partial_{i} P^{l-\varepsilon_{i}} \psi^{\mu}=-\sum_{a} \mu_{a} P^{l} \psi^{\mu+\varepsilon_{a}}+2 \sum_{i \neq j} \frac{l_{j}}{u_{i j}} P^{l-\varepsilon_{i}} \psi^{\mu}
$$

Indeed, differentiating we have

$$
\sum_{i} P^{l} \partial_{i} \psi^{\mu}=\sum_{a} \mu_{a} P^{l}\left(-\psi^{\mu+\varepsilon_{a}}+\psi^{\mu-\varepsilon_{a}} \sum_{i \neq j} \frac{1}{\left(u_{i}-z_{a}\right)\left(u_{j}-z_{a}\right)}\right) .
$$

The second term on the r.h.s. can be written as

$$
-\sum_{i \neq j} \frac{1}{\left(u_{i}-u_{j}\right)} \sum_{a} \mu_{a}\left(\frac{1}{\left(u_{i}-z_{a}\right)}-\frac{1}{\left(u_{j}-z_{a}\right)}\right) P^{l-\varepsilon_{i}-\varepsilon_{j}} \psi^{\mu-\varepsilon_{a}} .
$$

Applying formula (5.1.19) for the sum over $a$ we arrive at (5.2.4). Next we take the average over $l$ in (5.2.4), i.e., write $l=L \rho-\varepsilon_{E}$ and sum over $E \in E(r-1, m), r=L m-|\mu|$. The second term on the r.h.s. of (5.2.4) gives

$$
2 L \sum_{i \neq j} \frac{1}{u_{i j}} \varphi_{\mu}^{[L], i}-2 \sum_{i \neq j} \sum_{E \in E(r-1, m)} \frac{\left(\varepsilon_{E}\right)_{i}}{u_{i j}} \varphi_{\mu}^{L \rho-\varepsilon_{E}-\varepsilon_{i}} .
$$

The second term in the above is zero because taking the sum over $E$, in a way similar to (5.1.21), we end up with a symmetric sum over an antisymmetric quantity

$$
\sum_{i \neq j} \frac{1}{\left(u_{i}-u_{j}\right)} \varphi_{\mu}^{\ldots-\varepsilon_{i}-\varepsilon_{j}} \ldots=0
$$

Thus formula 5.2 .3 is proved. 
Now we proceed to prove the general non-thermal relations

$$
\sum_{a}\left(2 J_{a}-\sigma_{a}\right) \varphi_{\sigma+\varepsilon_{a}}^{\left(s, s^{\prime}\right)} \simeq 0
$$

or

$$
\sum_{a}\left(2 J_{a}-\sigma_{a}\right) I_{\sigma+\varepsilon_{a}}^{\left(s, s^{\prime}\right)}=0
$$

The derivation is not difficult but long, and we will shorten it at the cost of even greater proliferation of notation; thus let us set temporarily:

$$
\begin{aligned}
& \mathcal{T} \equiv \sum_{r}\left(\begin{array}{c}
R-1 \\
r
\end{array}\right) \sum_{|\alpha|=s-r-1}\left(\begin{array}{c}
\sigma \\
\alpha
\end{array}\right) \sum_{i, i^{\prime}} \frac{1}{\left(u_{i}-v_{i^{\prime}}\right)} \varphi_{\alpha}^{[1], i} \varphi_{\sigma-\alpha}^{[K]} \\
& \mathcal{T}^{\prime} \equiv \sum_{r}\left(\begin{array}{c}
R-1 \\
r
\end{array}\right) \sum_{|\alpha|=s-r}\left(\begin{array}{c}
\sigma \\
\alpha
\end{array}\right) \sum_{i, i^{\prime}} \frac{1}{\left(u_{i}-v_{i^{\prime}}\right)} \varphi_{\alpha}^{[1]} \varphi_{\sigma-\alpha}^{[K], i^{\prime}} \\
& \mathcal{X} \equiv \sum_{r}\left(\begin{array}{c}
R-1 \\
r
\end{array}\right) \sum_{|\alpha|=s-r-1}\left(\begin{array}{c}
\sigma \\
\alpha
\end{array}\right) \sum_{a}\left(2 J_{a}-\alpha_{a}\right) \varphi_{\alpha+\varepsilon_{a}}^{[1]} \varphi_{\sigma-\alpha}^{[K]}, \\
& \mathcal{X}^{\prime} \equiv \sum_{r}\left(\begin{array}{c}
R-1 \\
r
\end{array}\right) \sum_{|\alpha|=s-r}\left(\begin{array}{c}
\sigma \\
\alpha
\end{array}\right) \sum_{a}\left(2 J_{a}-\sigma_{a}+\alpha_{a}\right) \varphi_{\alpha}^{[1]} \varphi_{\sigma-\alpha+\varepsilon_{a}}^{[K]}, \\
& \mathcal{Z} \equiv \sum_{r}\left(\begin{array}{c}
R-1 \\
r
\end{array}\right)_{|\alpha|=s-r-1}\left(\begin{array}{c}
\sigma \\
\alpha
\end{array}\right) \sum_{a}\left(\sigma_{a}-\alpha_{a}\right) \varphi_{\alpha+\varepsilon_{a}}^{[1]} \varphi_{\sigma-\alpha}^{[K]}=\sum_{r}\left(\begin{array}{c}
R-1 \\
r
\end{array}\right) \sum_{|\alpha|=s-r}\left(\begin{array}{c}
\sigma \\
\alpha
\end{array}\right) \sum_{a} \alpha_{a} \varphi_{\alpha}^{[1]} \varphi_{\sigma-\alpha+\varepsilon_{a}}^{[K]} .
\end{aligned}
$$

From (5.1.16) we have the cohomological identity (derived in the same way as the (quasi)thermal relations):

$$
\mathcal{X}+\mathcal{X}^{\prime} \simeq 2\left(K \mathcal{T}-\mathcal{T}^{\prime}\right) .
$$

We obtain $\left(K \mathcal{T}-\mathcal{T}^{\prime}\right)$ if we apply the basic identity (5.1.20) to

$$
\sum_{r ; i, i^{\prime} ; a}\left(\begin{array}{c}
R-1 \\
r
\end{array}\right) \frac{1}{\left(u_{i}-v_{i^{\prime}}\right)}\left(\sum_{|\alpha|=s-r-1}\left(\begin{array}{c}
\sigma \\
\alpha
\end{array}\right) \frac{\left(\sigma_{a}-\alpha_{a}\right)}{\left(v_{i^{\prime}}-z_{a}\right)} \varphi_{\alpha}^{[1], i} \varphi_{\sigma-\alpha-\varepsilon_{a}}^{[K], i^{\prime}}-\sum_{|\alpha|=s-r}\left(\begin{array}{c}
\sigma \\
\alpha
\end{array}\right) \frac{\alpha_{a}}{\left(u_{i}-z_{a}\right)} \varphi_{\alpha-\varepsilon_{a}}^{[1], i} \varphi_{\sigma-\alpha}^{[K], i^{\prime}}\right) .
$$

On the other hand, using

$$
\frac{1}{\left(u_{i}-v_{i^{\prime}}\right)\left(v_{i^{\prime}}-z_{a}\right)}-\frac{1}{\left(u_{i}-v_{i^{\prime}}\right)\left(u_{i}-z_{a}\right)}=\frac{1}{\left(u_{i}-z_{a}\right)\left(v_{i^{\prime}}-z_{a}\right)}
$$

we obtain that $(5.2 .8)$ is equal to $\mathcal{Z}$.

Using a standard binomial coefficient formula $\left(\begin{array}{c}\sigma \\ \alpha\end{array}\right)+\left(\begin{array}{c}\sigma \\ \alpha-\varepsilon_{a}\end{array}\right)=\left(\begin{array}{c}\sigma+\varepsilon_{a} \\ \alpha\end{array}\right)$ in a multi-index guise we get from (5.2.7):

$$
(\mathcal{X}-\mathcal{Z})+\left(\mathcal{X}^{\prime}-\mathcal{Z}\right)=\sum_{a}\left(2 J_{a}-\sigma_{a}\right) \varphi_{\sigma+\varepsilon_{a}}^{\left(s, s^{\prime}\right)} \simeq 0
$$

i.e., the general non-thermal Aomoto-type relation. 


\subsection{Recursion relations}

The (quasi)thermal recursion relation is

$$
\sum_{a}\left(2 J_{a}-\mu_{a}\right) z_{a} \varphi_{\mu+\varepsilon_{a}}^{[L]}+c_{|\mu|}^{[L]} \varphi_{\mu}^{[L]} \simeq 0
$$

where

$$
c_{r}^{[L]}=2 \sum_{a=1}^{n-1} J_{a}-r-L m+1+K
$$

Of course, integrating (5.3.1) we obtain the recursion relation for the respective integrals. In order to prove the recursion relation we will have to establish the following identity

$$
\sum_{i} u_{i} \partial_{i} \varphi_{\mu}^{[L], i}=-\sum_{a} \mu_{a} z_{a} \varphi_{\mu+\varepsilon_{a}}^{[L]}-(|\mu|+(m-1) L) \varphi_{\mu}^{[L]}+2 L \sum_{i \neq j} \frac{u_{i}}{u_{i j}} \varphi_{\mu}^{[L], i} .
$$

Note that

$$
\frac{K}{L} \sum_{i} u_{i} \varphi_{\mu}^{[L], i} \partial_{i} \log \Phi^{[L]}=\sum_{a} 2 J_{a} \varphi_{\mu}^{[L]}+\sum_{a} 2 J_{a} z_{a} \varphi_{\mu+\varepsilon_{a}}^{[L]}-2 L \sum_{i \neq j} \frac{u_{i}}{u_{i j}} \varphi_{\mu}^{[L], i} .
$$

Therefore in the quasithermal case the cohomological identity (5.1.17) immediately translates into the recursion relation (5.3.1) when we combine (5.3.3) and (5.3.4). In the thermal case notice that

the 1.h.s. of (5.3.3) vanishes. Thus we can eliminate the last terms of (5.3.3) and (5.3.4) and again (5.1.17) gives us (5.3.1).

Now we proceed to prove (5.3.3). From the direct differentiation we have

$$
\begin{aligned}
& \sum_{i} u_{i} \partial_{i} P^{l-\varepsilon_{i}} \psi^{\mu}=\sum_{i} \sum_{a}\left(z_{a}+\left(u_{i}-z_{a}\right)\right) \mu_{a} P^{l}\left(\psi^{\mu-\varepsilon_{a}} \partial_{i} \psi_{a}\right)= \\
& \quad-\sum_{a} \mu_{a} z_{a} P^{l} \psi^{\mu-\varepsilon_{a}}\left(\psi_{a}^{2}-\sum_{i \neq j} \frac{1}{\left(u_{i}-z_{a}\right)\left(u_{j}-z_{a}\right)}\right)-\sum_{i} \sum_{a} \mu_{a} P^{l-\varepsilon_{i}} \frac{\psi^{\mu-\varepsilon_{a}}}{\left(u_{i}-z_{a}\right)}= \\
& \quad-\sum_{a} \mu_{a} z_{a} P^{l} \psi^{\mu+\varepsilon_{a}}+\sum_{a} \mu_{a} z_{a} P^{l} \psi^{\mu+\varepsilon_{a}} \sum_{i \neq j} \frac{1}{\left(u_{i}-z_{a}\right)\left(u_{j}-z_{a}\right)}-\sum_{i}\left(l_{i}-1\right) P^{l-\varepsilon_{i}} \psi^{\mu}
\end{aligned}
$$

where to obtain the third term of (5.3.5) we have used (5.1.19). Substitute $z_{a}=-\left(u_{i}-z_{a}\right)+u_{i}$, in the second term of (5.3.5) and rewrite this term as follows

$$
-\sum_{i \neq j} \sum_{a} \mu_{a} P^{l-\varepsilon_{i}} \frac{\psi^{\mu-\varepsilon_{a}}}{\left(u_{j}-z_{a}\right)}+\sum_{i \neq j} \frac{u_{i}}{u_{i j}} \sum_{a} \mu_{a}\left(\frac{1}{\left(u_{j}-z_{a}\right)}-\frac{1}{\left(u_{i}-z_{a}\right)}\right) P^{l-\varepsilon_{i}-\varepsilon_{j}} \psi^{\mu-\varepsilon_{a}} .
$$

Applying (5.1.19) to the above we get

$$
-\sum_{i \neq j} l_{j} P^{l-\varepsilon_{i}} \psi^{\mu}+2 \sum_{i \neq j} \frac{u_{i}}{u_{i j}}\left(l_{j} P^{l-\varepsilon_{i}}-l_{i} P^{l-\varepsilon_{j}}\right) \psi^{\mu}=-2 \sum_{i \neq j} l_{j} P^{l-\varepsilon_{i}} \psi^{\mu}+2 \sum_{i \neq j} \frac{u_{i}}{u_{i j}} l_{j} P^{l-\varepsilon_{i}} \psi^{\mu} .
$$

Now substitute back (5.3.6) for the second term of (5.3.5). and rewrite $-\sum_{i}\left(l_{i}-1\right) P^{l-\varepsilon_{i}}-$ $2 \sum_{i \neq j} l_{j} P^{l-\varepsilon_{i}}$ as $-|\mu| \sum_{i} P^{l-\varepsilon_{i}}-\sum_{i \neq j} l_{j} P^{l-\varepsilon_{i}}$, taking into account that $|\mu|=|l|-1$ in this case. Thus we obtain the following identity:

$$
\sum_{i} u_{i} \partial_{i} P^{l-\varepsilon_{i}} \psi^{\mu}=-\sum_{a} \mu_{a} z_{a} P^{l} \psi^{\mu+\varepsilon_{a}}-|\mu| \sum_{i} P^{l-\varepsilon_{i}} \psi^{\mu}+\sum_{i \neq j}\left(\frac{2 u_{i}}{u_{i j}}-1\right) l_{j} P^{l-\varepsilon_{i}} \psi^{\mu} .
$$


If we perform the averaging over $l$, i.e., set $l=L \rho-\varepsilon_{E}$ and sum over $E$ we arrive at (5.3.3) and thus we have derived the (quasi)thermal recursion relations.

The proof of the general recursion relation

$$
\sum_{a}\left(2 J_{a}-\sigma_{a}\right) z_{a} \varphi_{\sigma+\varepsilon_{a}}^{\left(s, s^{\prime}\right)}+c_{|\sigma|} \varphi_{\sigma}^{\left(s, s^{\prime}\right)} \simeq 0
$$

where

$$
c_{|\sigma|} \equiv 2 J_{n}+1+K+S-|\sigma|=\sum_{a=1}^{n} J_{a}+1+K-|\sigma|=2 \sum_{a=1}^{n-1} J_{a}+1+K-S-|\sigma|,
$$

though somewhat more involved, is nevertheless analogous to the derivation of the general nonthermal linear relation in the previous subsection and we will skip it.

\subsection{Differential equations}

In this section we will show that the integrals (5.1.8) satisfy the following system of differential equations

$$
\begin{aligned}
& -K \partial_{a} I_{\mu}^{[L]}=(L m-|\mu|)\left(2 J_{a}-\mu_{a}\right) I_{\mu+\varepsilon_{a}}^{[L]} \\
& \quad-\sum_{b(\neq a)} \frac{1}{\left(z_{a}-z_{b}\right)}\left[\mu_{a}\left(2 J_{b}-\mu_{b}\right)\left(I_{\mu}^{[L]}-I_{\mu-\varepsilon_{a}+\varepsilon_{b}}^{[L]}\right)+\mu_{b}\left(2 J_{a}-\mu_{a}\right)\left(I_{\mu}^{[L]}-I_{\mu+\varepsilon_{a}-\varepsilon_{b}}^{[L]}\right)\right]
\end{aligned}
$$

or, written in terms of a cohomological identity for $m$-forms:

$$
\begin{aligned}
-K & D_{a} \varphi_{\mu}^{[L]} \simeq(L m-|\mu|)\left(2 J_{a}-\mu_{a}\right) \varphi_{\mu+\varepsilon_{a}}^{[L]} \\
& \left.-\sum_{b(\neq a)} \sum_{i} \frac{1}{\left(u_{i}-z_{a}\right)\left(u_{i}-z_{b}\right)}\left[\mu_{a}\left(2 J_{b}-\mu_{b}\right) \varphi_{\mu-\varepsilon_{a}}^{[L], i}-\mu_{b}\left(2 J_{a}-\mu_{a}\right) \varphi_{\mu-\varepsilon_{b}}^{[L], i}\right)\right]
\end{aligned}
$$

where $D_{a}=\partial_{a}+\partial_{a} \log \Phi^{[L]}$.

Before we proceed let us introduce the notation

$$
\mathcal{M}_{a b}=\sum_{i} \frac{1}{\left(u_{i}-z_{a}\right)\left(u_{i}-z_{b}\right)} \varphi_{\mu-\varepsilon_{a}}^{[L], i} .
$$

If $a \neq b$ we can rewrite it as follows

$$
\mathcal{M}_{a b}=\frac{1}{\left(z_{a}-z_{b}\right)}\left(\varphi_{\mu}^{[L]}-\varphi_{\mu-\varepsilon_{a}+\varepsilon_{b}}^{[L]}\right)
$$

and in particular we see that integrating (5.4.2) we obtain (5.4.1). One immediately obtains (5.4.2) if the following identities are established (recall that we denote $\left.\psi_{a}=\sum_{i}\left(\left(u_{i}-z_{a}\right)\right)^{-1}\right)$ :

$$
\begin{aligned}
& -K D_{a} \varphi_{\mu}^{[L]}=2 J_{a} L \psi_{a} \varphi_{\mu}^{[L]}-\mu_{a} K \mathcal{M}_{a a} \\
& \sum_{b} \mu_{b} \mathcal{M}_{b a}-L \psi_{a} \varphi_{\mu}^{[L]}+(L m-|\mu|) \varphi_{\mu+\varepsilon_{a}}^{[L]}=0 \\
& \sum_{b}\left(2 J_{b}-\mu_{b}\right) \mathcal{M}_{a b}+L \psi_{a} \varphi_{\mu}^{[L]}-K \mathcal{M}_{a a} \simeq 0
\end{aligned}
$$


The first equation follows directly by differentiating, the second follows immediately from our basic relation (5.1.20) while the cohomological identity (5.4.5) we will derive from the cohomological identity

$$
D \sum_{i} \frac{1}{\left(u_{i}-z_{a}\right)} \varphi_{\mu-\varepsilon_{a}}^{[L], i} \prod_{j(\neq i)} d u_{j} \simeq 0 .
$$

To prove (5.4.5) we will establish

$$
\sum_{i} \frac{1}{\left(u_{i}-z_{a}\right)} \partial_{i} \varphi_{\mu-\varepsilon_{a}}^{[L], i}=-\sum_{b}\left(\mu-\varepsilon_{a}\right)_{b} \mathcal{M}_{a b}+L \sum_{i \neq j} \frac{1}{\left(u_{i}-u_{j}\right)}\left(\frac{1}{\left(u_{i}-z_{a}\right)}+\frac{1}{\left(u_{j}-z_{a}\right)}\right) \varphi_{\mu-\varepsilon_{a}}^{[L], i} .
$$

In the thermal case the 1.h.s. of (5.4.7) is zero, and adding (5.4.7) multiplied by $K^{-1}$ to (5.4.6) we obtain (5.4.5). In the quasithermal case, substituting (5.4.7) into (5.4.6) we obtain (5.4.5). So let us now derive (5.4.7). Differentiating we obtain

$$
\begin{aligned}
\sum_{i} & \frac{1}{\left(u_{i}-z_{a}\right)} P^{l} \partial_{i} \psi^{\mu-\varepsilon_{a}}=-\sum_{i} \sum_{b} \frac{\left(\mu-\varepsilon_{a}\right)_{b}}{\left(u_{i}-z_{a}\right)\left(u_{i}-z_{b}\right)} P^{l-\varepsilon_{i}} \psi^{\mu-\varepsilon_{a}} \\
& +\sum_{i \neq j} \sum_{b} \frac{\left(\mu-\varepsilon_{a}\right)_{b}}{u_{i j}}\left(\frac{1}{\left(u_{j}-z_{b}\right)}-\frac{1}{\left(u_{i}-z_{b}\right)}\right) P^{l-\varepsilon_{i}} \frac{\psi^{\mu-\varepsilon_{a}-\varepsilon_{b}}}{\left(u_{i}-z_{a}\right)} .
\end{aligned}
$$

Apply to the second term of the r.h.s. our identity (5.1.19) to obtain

$$
\sum_{i} \frac{1}{\left(u_{i}-z_{a}\right)} \partial_{i} \varphi_{\mu-\varepsilon_{a}}^{l-\varepsilon_{i}}=-\sum_{b} \sum_{i} \frac{\left(\mu-\varepsilon_{a}\right)_{b}}{u_{i j}} \varphi_{\mu-\varepsilon_{a}}^{l-\varepsilon_{i}}+\sum_{i \neq j} \frac{l_{j}}{u_{i j}}\left(\frac{1}{\left(u_{j}-z_{b}\right)}+\frac{1}{\left(u_{i}-z_{b}\right)}\right) \varphi_{\mu-\varepsilon_{a}}^{l-\varepsilon_{i}} .
$$

Now average over $l$ in the above. As in the previous cases, for part of the second term we get a zero because of a symmetric sum over an antisymmetric quantity and this completes the derivation of (5.4.7) and, as already discussed, also of (5.4.2).

The general non-thermal differential equation is

$$
\begin{aligned}
& -K D_{a} I_{\sigma}^{\left(s, s^{\prime}\right)}=(S-|\sigma|)\left(2 J_{a}-\sigma_{a}\right) I_{\sigma+\varepsilon_{a}}^{\left(s, s^{\prime}\right)} \\
& \quad-\sum_{b(\neq a)} \frac{1}{\left(z_{a}-z_{b}\right)}\left(\sigma_{a}\left(2 J_{b}-\sigma_{b}\right)\left(I_{\sigma}^{\left(s, s^{\prime}\right)}-I_{\sigma-\varepsilon_{a}+\varepsilon_{b}}^{\left(s, s^{\prime}\right)}\right)+\sigma_{b}\left(2 J_{a}-\sigma_{a}\right)\left(I_{\sigma}^{\left(s, s^{\prime}\right)}-I_{\sigma+\varepsilon_{a}-\varepsilon_{b}}^{\left(s, s^{\prime}\right)}\right) .\right.
\end{aligned}
$$

Its derivation is again similar to the derivation of the general non-thermal linear and recursion relations. Using the complete non-thermal local system we obtain, in analogy with the (quasi)thermal case (5.4.2), the following cohomological identity

$$
\begin{aligned}
& -K D_{a} \phi_{\alpha, \beta} \simeq 2\left(K \alpha_{a} \mathcal{T}_{\alpha-\varepsilon_{a}, \beta}-\beta_{a} \mathcal{T}_{\alpha, \beta-\varepsilon_{a}}^{\prime}\right)+r\left(2 J_{a}-\alpha_{a}\right) \phi_{\alpha+\varepsilon_{a}, \beta}+(R-r)\left(2 J_{a}-\beta_{a}\right) \phi_{\alpha, \beta+\varepsilon_{a}} \\
& -\sum_{b(\neq a)}\left(\alpha_{a}\left(2 J_{b}-\alpha_{b}\right) \mathcal{M}_{a b}-\alpha_{b}\left(2 J_{a}-\alpha_{a}\right) \mathcal{M}_{b a}\right)-\sum_{b(\neq a)}\left(\beta_{a}\left(2 J_{b}-\beta_{b}\right) \mathcal{M}_{a b}^{\prime}-\beta_{b}\left(2 J_{a}-\beta_{a}\right) \mathcal{M}_{b a}^{\prime}\right)
\end{aligned}
$$

where $\alpha+\beta=\sigma$ and this time we have denoted

$$
\mathcal{M}_{a b}=\frac{1}{\left(z_{a}-z_{b}\right)}\left(\varphi_{\alpha}^{[1]} \varphi_{\beta}^{[K]}-\varphi_{\alpha-\varepsilon_{a}+\varepsilon_{b}}^{[1]} \varphi_{\beta}^{[K]}\right), \quad \mathcal{M}_{a b}^{\prime}=\frac{1}{\left(z_{a}-z_{b}\right)}\left(\varphi_{\alpha}^{[1]} \varphi_{\beta}^{[K]}-\varphi_{\alpha}^{[1]} \varphi_{\beta-\varepsilon_{a}+\varepsilon_{b}}^{[K]}\right),
$$




$$
\mathcal{T}_{\alpha-\varepsilon_{a}, \beta}=\sum_{i, i^{\prime}} \frac{1}{\left(u_{i}-v_{i^{\prime}}\right)\left(u_{i}-z_{a}\right)} \varphi_{\alpha-\varepsilon_{a}}^{[1], i} \varphi_{\beta}^{[K]}, \quad \mathcal{T}_{\alpha, \beta-\varepsilon_{a}}=\sum_{i, i^{\prime}} \frac{1}{\left(u_{i}-v_{i^{\prime}}\right)\left(v_{i^{\prime}}-z_{a}\right)} \varphi_{\alpha}^{[1]} \varphi_{\beta-\varepsilon_{a}}^{[K], i^{\prime}}
$$

and again $|\alpha|=s-r$, and $R=S-|\sigma|=K s^{\prime}-|\beta|+r$. To compare with (5.4.8) we have to multiply (5.4.9) by $\left(\begin{array}{c}\sigma \\ \alpha\end{array}\right)$ and sum over $\alpha$. To obtain the term $2\left(K \mathcal{T}-\mathcal{T}^{\prime}\right)$ of $(5.4 .9)$ apply the basic identity (5.1.20) to

$$
\sum_{\alpha}\left(\begin{array}{c}
S-|\sigma| \\
s-|\alpha|
\end{array}\right)\left(\begin{array}{c}
\sigma \\
\alpha
\end{array}\right) \sum_{i i^{\prime} ; b} \frac{1}{\left(u_{i}-v_{i^{\prime}}\right)}\left(\frac{\alpha_{a} \beta_{b} \varphi_{\alpha-\varepsilon_{a}}^{[1], i} \varphi_{\beta-\varepsilon_{b}}^{[K], i^{\prime}}}{\left(v_{i^{\prime}}-z_{b}\right)\left(u_{i}-z_{a}\right)}-\frac{\alpha_{b} \beta_{a} \varphi_{\alpha-\varepsilon_{b}}^{[1], i} \varphi_{\beta-\varepsilon_{a}}^{[K], i^{\prime}}}{\left(u_{i}-z_{b}\right)\left(v_{i^{\prime}}-z_{a}\right)}\right) .
$$

Substituting in the above the following identity,

$$
\begin{gathered}
\frac{1}{\left(u_{i}-v_{i^{\prime}}\right)\left(u_{i}-z_{a}\right)\left(v_{i^{\prime}}-z_{b}\right)}-\frac{1}{\left(u_{i}-v_{i^{\prime}}\right)\left(v_{i^{\prime}}-z_{a}\right)\left(u_{i}-z_{b}\right)} \\
=\frac{1}{\left(z_{a}-z_{b}\right)}\left(\frac{-1}{\left(u_{i}-z_{a}\right)\left(v_{i^{\prime}}-z_{b}\right)}+\frac{1}{\left(u_{i}-z_{b}\right)\left(v_{i^{\prime}}-z_{a}\right)}+\frac{1}{\left(u_{i}-z_{a}\right)\left(v_{i^{\prime}}-z_{a}\right)}+\frac{1}{\left(u_{i}-z_{b}\right)\left(v_{i^{\prime}}-z_{b}\right)}\right)
\end{gathered}
$$

and after some algebra we obtain (5.4.8).

\section{Discussion}

We have found solutions of the KZ equations of the $A_{1}^{(1)}$ WZNW conformal theory for arbitrary level $k \neq-2$ and some isospin values corresponding to infinite dimensional representations of $A_{1}-$ namely the isospins of the type in (1.3) $, J_{j, j^{\prime}}, 2 j, 2 j^{\prime} \in \mathbb{Z}_{+}-$although we were mainly interested in the "minimal subset" described by rational $k$ and by the domain in (1.4), (1.5).

These are precisely the values which parametrize according to (1.6), (1.7), all reducible Virasoro Verma modules. Reducibility implies differential (BPZ) equations for the conformal blocks of primary fields. Our results show that these blocks can be "imbedded" in a set of generalized hypergeometric integrals which satisfy a first-order linear differential system of equations and serve as coefficients of the infinite $(x-z)$ - expansion representing the correlators of the $A_{1}^{(1)}$ WZNW model. We have given strong arguments that the KZ equation, written as an infinite - in general - system for the coefficients in the $(x-z)$ - expansion, reduces to a finite system from which one can recover the BPZ equation for the Virasoro correlator. Yet it remains a rather difficult technical problem to do this explicitly in general - either by exploiting the existing finite system in [22], or by taking into account that the solutions of the KZ equation satisfy also $A_{1}^{(1)}$ null vector decoupling equations.

This is related to the problem of constructing the expressions for the singular vectors of the Virasoro Verma modules "via quantum Hamiltonian reduction". The simpler thermal (and quasithermal) cases discussed above shed some light on the relation of our construction and the approach in [31] where an algorithm for obtaining all Virasoro singular vectors is proposed (see also the recent paper [35], which we received while this work was in process.) In particular in [31] the analogue of our infinite system in the general non-thermal case is avoided, using the fusion procedure.

The peculiarities of the rational level cases were only briefly touched in this paper. The rational level theories require a further and more thorough study - especially in connection with the description of the relevant twisted homology and cohomology groups. 
In our " $(x-z)$ " - expansion of the correlators and the proposed solution in terms of "meromorphic" forms we have departed from bosonization. This raises the problem of finding a proper operator language behind the construction.

There is an important open problem which we have not touched so far, namely the understanding of the isospin $s l_{I}(2, \mathscr{C})$ tensor product decomposition of the correlation functions. This requires yet another expansion, extending the representation used in [14] to the case of non-integer isospins $2 J_{a}$.

Finally let us point out that the generalization of our results to the case of higher rank algebras is also possible.

\section{Acknowledgements}

The authors would like to thank L. Alvarez-Gaumé, P. Bouwknegt, L. Dạbrowski, V.K. Dobrev, Vl.S. Dotsenko, V.G. Kac, and C. Reina for useful discussions. V.B.P. acknowledges the hospitality and the financial support of INFN and SISSA.

\section{A Appendix}

Here we will establish an algorithm determining E. The notation is the same as in Section 4.2. Consider the $(+1),(0),(-1), \ldots$ diagonals of $(4.2 .9)$. From this equation we can determine successively the matrix elements of $\mathrm{E}^{(0)}, \mathrm{E}^{(-1)}, \mathrm{E}^{(-2)}, \ldots$.

First consider the $(+1)$ diagonal of $(4.2 .9)$. We have $\left(\mathrm{E}^{(0)} \mathrm{F}^{(1)}\right)_{i i+1}=\left(\hat{\mathrm{F}}^{(1)} \mathrm{E}^{(0)}\right)_{i i+1}$ or

$$
\mathrm{E}_{i+1}^{(0)}=\mathrm{E}_{i}^{(0)} \mathrm{F}_{i}^{(1)} / \hat{\mathrm{F}}_{i}^{(1)}
$$

while from (4.2.7) we have

$$
\mathrm{E}_{0}^{(0)}=1
$$

thus

$$
\mathrm{E}_{i+1}^{(0)}=\prod_{k=0}^{i}\left(\frac{\mathrm{F}_{k}^{(1)}}{\hat{\mathrm{F}}_{k}^{(1)}}\right) \quad i=0,1,2 \ldots
$$

and all $\mathrm{E}_{i}^{(0)}$ are constants (i.e., no $z$ dependence).

From the $(-n+1)$ diagonal of $(4.2 .9)$ we get a simple recursion for the elements of $\mathrm{E}^{(-n)}$ of the form:

$$
\mathrm{E}_{j+1}^{(-n)}=\mathcal{D}_{j}^{(n)} \mathrm{E}_{j}^{(-n)}+\mathcal{C}_{j}^{(n)}
$$

The initial condition

$$
\mathrm{E}_{n-1}^{(-n)}=0
$$

is obvious if we note that the indices of our matrices $E$ and $F$ run over the non-negative integers. The solution of this recursion is

$$
\mathrm{E}_{j+1}^{(-n)}=\mathcal{C}_{j}^{(n)}+\sum_{i=0}^{j-n} \mathcal{D}_{j}^{(n)} \mathcal{D}_{j-1}^{(n)} \ldots \mathcal{D}_{j-i}^{(n)} \mathcal{C}_{j-i-1}^{(n)}, \quad j=n, n+1, n+2, \ldots
$$


Let us consider the first few values of $n$. The (0) diagonal of (4.2.9) gives:

$$
\mathrm{E}^{(0)}\left(\mathrm{F}^{(0)}+\partial\right)+\mathrm{E}^{(-1)} \mathrm{F}^{(1)}=\left(\hat{\mathrm{F}}^{(0)}+\partial\right) \mathrm{E}^{(0)}+\hat{\mathrm{F}}^{(1)} \mathrm{E}^{(-1)} .
$$

Because $\mathrm{E}^{(0)}$ is a constant matrix, the terms with $\partial$ cancel. We get a recursion of the form (A.2) with $\mathcal{D}_{i}^{(1)}=\mathrm{F}_{i-1}^{(1)} / \hat{\mathrm{F}}_{i}^{(1)}$ and $\mathcal{C}_{i}^{(1)}=\mathrm{E}_{i}^{(0)}\left(\mathrm{F}_{i}^{(0)}-\hat{\mathrm{F}}_{i}^{(0)}\right) / \hat{\mathrm{F}}_{i}^{(1)}$ and initial condition $\mathrm{E}_{0}^{(-1)}=0$ (it is easy to note that in fact we have $\left.\mathrm{E}_{1}^{(-1)}=0\right)$.

From the $(-1)$ diagonal of 4.2 .9 we obtain:

$$
\mathrm{E}^{(0)} \mathrm{F}^{(-1)}+\mathrm{E}^{(-1)} \mathrm{F}^{(0)}+\mathrm{E}^{(-2)} \mathrm{F}^{(1)}=\hat{\mathrm{F}}^{(-1)} \mathrm{E}^{(0)}+\hat{\mathrm{F}}^{(0)} \mathrm{E}^{(-1)}+\left(\partial \mathrm{E}^{(-1)}\right)+\hat{\mathrm{F}}^{(1)} \mathrm{E}^{(-2)}
$$

which gives a recursion for $\mathrm{E}^{(-2)}$ with

$$
\mathcal{D}_{i}^{(2)}=\frac{\mathrm{F}_{i-2}^{(1)}}{\hat{\mathrm{F}}_{i}^{(1)}}, \quad \mathcal{C}_{i}^{(2)}=\frac{1}{\hat{\mathrm{F}}_{i}^{(1)}}\left[\mathrm{E}_{i}^{(0)} \mathrm{F}_{i}^{(-1)}-\mathrm{E}_{i-1}^{(0)} \hat{\mathrm{F}}_{i}^{(-1)}+\mathrm{E}_{i}^{(-1)}\left(\mathrm{F}_{i-1}^{(0)}-\hat{\mathrm{F}}_{i}^{(0)}\right)-\left(\partial \mathrm{E}_{i}^{(-1)}\right)\right] .
$$

Obviously this is the most general case, i.e. for $\mathrm{E}^{(-k)}$ with $k=2,3, \ldots$ we have

$$
\mathcal{D}_{i}^{(k)}=\frac{\mathrm{F}_{i-k}^{(1)}}{\hat{\mathrm{F}}_{i}^{(1)}}, \quad \mathcal{C}_{i}^{(k)}=\frac{1}{\hat{\mathrm{F}}_{i}^{(1)}}\left[\mathrm{E}_{i}^{(-k+2)} \mathrm{F}_{i-k+2}^{(-1)}-\mathrm{E}_{i-1}^{(-k+2)} \hat{\mathrm{F}}_{i}^{(-1)}+\mathrm{E}_{i}^{(-k+1)}\left(\mathrm{F}_{i-k+1}^{(0)}-\hat{\mathrm{F}}_{i}^{(0)}\right)-\left(\partial \mathrm{E}_{i}^{(-k+1)}\right)\right] .
$$

Thus we have an algorithm to determine the matrix $\mathrm{E}$.

Let us give the answer for the first few entries:

$$
\begin{aligned}
\mathrm{E}_{1}^{(0)}= & 0, \quad \mathrm{E}_{i}^{(0)}=\frac{\mathrm{F}_{i-1}^{(1)} \ldots \mathrm{F}_{0}^{(1)}}{\hat{\mathrm{F}}_{i-1}^{(1)} \ldots \hat{\mathrm{F}}_{0}^{(1)}} i=1,2, \ldots, \\
\mathrm{E}_{1}^{(-1)}= & 0, \quad \mathrm{E}_{2}^{(-1)}=\frac{\mathrm{F}_{0}^{(1)}\left(\mathrm{F}_{1}^{(0)}-\hat{\mathrm{F}}_{1}^{(0)}\right)}{\hat{\mathrm{F}}_{1}^{(1)} \hat{\mathrm{F}}_{0}^{(1)}}, \\
\mathrm{E}_{2}^{(-2)=} & \frac{\mathrm{F}_{0}^{(1)} \mathrm{F}_{1}^{(-1)}-\hat{\mathrm{F}}_{1}^{(-1)} \hat{\mathrm{F}}_{0}^{(1)}}{\hat{\mathrm{F}}_{1}^{(1)} \hat{\mathrm{F}}_{0}^{(1)}}, \\
\mathrm{E}_{3}^{(-2)=} & \frac{\mathrm{F}_{0}^{(1)}}{\hat{\mathrm{F}}_{2}^{(1)} \hat{\mathrm{F}}_{1}^{(1)} \hat{\mathrm{F}}_{0}^{(1)}}\left(\mathrm{F}_{0}^{(1)} \mathrm{F}_{1}^{(-1)}-\hat{\mathrm{F}}_{0}^{(1)} \hat{\mathrm{F}}_{1}^{(-1)}+\mathrm{F}_{1}^{(1)} \mathrm{F}_{2}^{(-1)}\right. \\
& \left.-\hat{\mathrm{F}}_{1}^{(1)} \hat{\mathrm{F}}_{2}^{(-1)}+\left(\mathrm{F}_{1}^{(0)}-\hat{\mathrm{F}}_{1}^{(0)}\right)\left(\mathrm{F}_{1}^{(0)}-\hat{\mathrm{F}}_{2}^{(0)}\right)-\partial\left(\mathrm{F}_{1}^{(0)}-\hat{\mathrm{F}}_{1}^{(0)}\right)\right) .
\end{aligned}
$$

And at the end we write down some very explicit formulas

$$
\begin{aligned}
\mathrm{E}_{2}^{(0)} & =\frac{\left(2 J_{2}-1\right)(s-1)}{\left(2 J_{2}-K\right)(s-K)}, \\
\mathrm{E}_{2}^{(-1)} & =\frac{(K-1)}{\left(2 J_{2}-K\right)(s-K)}\left(\frac{2 J_{1}+2 J_{2}}{z}+\frac{2 J_{2}+2 J_{3}}{z-1}\right), \\
\mathrm{E}_{2}^{(-2)} & =\frac{(K-1)\left(J_{1}+J_{2}+J_{3}+J_{4}+1+K\right)}{\left(2 J_{2}-K\right)(s-K) z(z-1)} .
\end{aligned}
$$




\section{References}

[1] V.G. Knizhnik and A.B. Zamolodchikov, Nucl. Phys. B247 (1984) 83.

[2] A.M. Polyakov, Mod. Phys Lett. A2 (1987) 893, V.G. Knizhnik, A.M. Polyakov and A.B. Zamolodchikov, Mod. Phys. Lett. A3 (1988) 819.

[3] B.L. Feigin and D.B. Fuchs, Funk. Anal. Prilpzh. 16, No 2 (1982) 47.

[4] V.G. Kac and M. Wakimoto, Proc. Natl. Acad. Sci. USA 85 (1988) 4956.

[5] D. Bernard and G. Felder, Comm. Math. Phys. 127 (1990) 145.

[6] V. Drinfeld and V. Sokolov, J. Sov. Math. 30 (1984) 1975.

[7] M. Bershadsky and H. Ooguri, Comm. Math. Phys. 126 (1989) 49, B. Feigin and E. Frenkel, Phys. Lett. B246 (1990) 75.

[8] S. Mukhi and S. Panda, Nucl. Phys. B338 (1990) 263.

[9] V.G. Kac and M. Wakimoto, Acta Appl. Math. 21 (1990) 3.

[10] P. Furlan, R. Paunov, A.Ch. Ganchev and V.B. Petkova, Phys. Lett. B267 (1991) 63.

[11] A.B. Zamolodchikov and V.A. Fateev, Sov. J. Nucl. Phys. 43 (1986) 657.

[12] A. Tsuchyia and Y. Kanie, Lett. Math. Phys. 13 (1987) 303; Adv. Stud. Pure Math. 16 (1988) 297; Errata ibid 19 (1990) 675.

[13] T. Kohno, Ann. Inst. Fourier 37, 4 (1987) 139.

[14] P. Christe and R. Flume, Nucl. Phys. B282 (1987) 466, P. Christe, PhD Thesis, Bonn University (1986).

[15] E. Date, M. Jimbo, A. Matsuo and T. Miwa, in: Yang-Baxter Equations, Conformal Invariance and Integrability in Statistical Mechanics and Field Theory, World Scientific (1989).

[16] A.Gerasimov, A.Morozov, M. Olshanetski, A. Marshakov, and S. Shatashvili, Int. J. Mod. Phys. A5 (1990) 2495,

B.L. Feigin and E.V. Frenkel, in: V.G. Knizhnik Memorial volume, eds. L. Brink et al, World Scientific, Singapore, 1990, Vl.S. Dotsenko, Nordita preprint, 89/54 P (1989), (to appear in Nucl. Phys. B).

[17] V.V. Schechtman and A.N. Varchenko, Lett. Math. Phys. 20 (1990) 279; Inven. Math. 106 (1991) 139.

[18] A. Matsuo, Comm. Math. Phys. 134 (1990) 65, G. Kuroki, Comm. Math. Phys. 142 (1991) 511, F. Falceto, K. Gawedzki and A. Kupiainen, Bures-sur-Yvette preprint, IHES/P/91/17, 1991, H. Awata, A. Tsuchiya and Y. Yamada, Tsukuba preprint, KEK-TH-286, 1991.

[19] V.S. Dotsenko and V.A. Fateev, Nucl. Phys. B240 (1984) 312; B251 (1985) 691. 
[20] A. Tsuchyia and Y. Kanie, Publ. RIMS 22 (1986) 259.

[21] G. Felder, Nucl. Phys. B317 (1989) 215.

[22] K. Aomoto, J. Math. Soc. Japan 39 (1987) 191.

[23] V.A. Vasil'ev, I.M. Gel'fand and A.V. Zelevinskii, Funct. Anal. Prilozhen. 21 (1987) 19-31.

[24] Vl.S. Dotsenko, Santa Barbara preprint, NSF-ITP-90-148, (1990).

[25] A. Belavin, A. Polyakov and A. Zamolodchikov, Nucl. Phys. B241 (1984) 333.

[26] G. Felder, "Minimal models on Riemann surface", in: Proc. of the ICTP conference on "New developments in conformal field theory", S. Ranjbar-Daemi and J.-B. Zuber (eds.), World Scientific (1990),

G. Felder and R. Silvotti, Phys. Lett. B231 (1989) 411.

[27] C. Gomez and G. Sierra, Nucl. Phys. B352 (1991) 791.

[28] B.L. Feigin, V.V. Schechtman and A.N. Varchenko, Lett. Math. Phys. 20 (1990) 291.

[29] L. Benoit and Y. Saint-Aubin, Phys. Lett. B215 (1988) 517.

[30] T.S. Choi, I.G. Koh, B.C. Park and H.J. Shin, Phys. Lett. B233 (1989) 163.

[31] M. Bauer, Ph. Di Francesco, C. Itzykson and J.-B. Zuber, Phys. Lett. B260 (1991) 323; Nucl. Phys. B362 (1991) 515.

[32] V. G. Kac and D. A. Kazhdan, Adv. Math. 34 (1979) 97.

[33] F.G. Malikov, B.L. Feigin and D.B. Fuks, Funct. Anal. Prilozhen. 20, no. 2 (1987) 25.

[34] V.K. Dobrev, Lett. Math. Phys. 22 (1991) 251.

[35] M. Bauer and N. Sochen, Saclay preprint, SPhT /91-117. 